\title{
The Decoherence Time in High Energy Heavy Ion Collisions
}

\author{
Berndt Müller \\ Department of Physics, Duke University, Durham, North Carolina 27708 \\ Andreas Schäfer \\ Institut für Theoretische Physik, Universität Regensburg, D-93040 Regensburg, Germany
}

(Dated: May 2, 2022)

\begin{abstract}
We calculate the decoherence time of the ground state wave function of a nucleus in a high energy heavy ion collision. We define this time as the decay time of the ratio $\operatorname{Tr} D^{2} /(\operatorname{Tr} D)^{2}$ of traces of the density matrix $D$. We find that this time is smaller or equal to $1 / Q_{s}$, where the saturation scale $Q_{s}$ is defined within the color glass condensate model of parton saturation. Our result supports the notion that the extremely rapid entropy production deduced for the early stage of heavy ion collisions at collider energies is to a large extent caused by the decoherence of the initial-state wave functions.
\end{abstract}

PACS numbers: 25.75.-q,13.85.-t

\section{I. INTRODUCTION}

The physics program of the Relativistic Heavy Ion Collider (RHIC) has produced many intriguing results and posed a number of unexplained problems. One central question that has emerges is: How can hydrodynamical behaviour, implying local thermal equilibration and complete decoherence of the initial state, occur on a time scale which is considerably shorter than $1 \mathrm{fm} / c$ ? [1, 2].

In an earlier article [3] we showed that the entropy per rapidity interval produced by decoherence alone is proportional to $\left(R Q_{s}\right)^{2}$, where $R$ is the nuclear radius and $Q_{s}$ is the gluon saturation scale [4]. The resulting entropy per unit rapidity interval is of the order $1000-2000$, which amount to a substantial fraction of the total produced entropy. We than argued that the decoherence time has to be of order $1 / Q_{s}$, as this is the natural scale of the process. The purpose of the present article is to substantiate this claim by means of a quantitative calculation.

The fact that entropy produced by decoherence can play an important role in high-energy heavy-ion collisions was to the best of our knowledge first discussed in [5].

Our article is organized as follows: In Section II we explain how we attack the problem and how we define the decoherence time. In Section III we describe the calculation of the density matrix for gluons, which undergo a hard scattering process, in detail. Our calculation is based on work by Kovchegov and Mueller [10, 11] on gluon production in heavy ion collisions. We generalize their derivation of probabilities to the level of density matrices. In Section IV we calculate the decoherence time using the results from Section III.

\section{STRATEGY}

Our goal is to obtain an estimate of the decoherence time of the gluon distribution in a large nucleus (1), when it is hit by another very energetic large nucleus (2). In principle, the decoherence process is encoded in the time evolution of the density matrix $\hat{D}$ in a very simple manner: For vanishing off-diagonal matrix elements the system is completely decoherent. In our case the dominant degrees of freedom are the gluons and thus the relevant density matrix is that of the gluons in nucleus 1. Decoherence thus manifests itself in a gradual disappearance of its off-diagonal elements $D_{k_{1}, k_{1}^{\prime}}$ with the momenta $k_{1} \neq k_{1}^{\prime}$.

This sounds simple enough, but actually calculating the time evolution of off-diagonal elements in a complex multiparticle state is an extremely difficult task [6, 7]. Therefore, one has studied so far mainly very simple toy models, like one harmonic oscillator in a termal bath of other harmonic oscillators, or rather specific situations, like neutrino oscillations [8].

Luckily, also the situation encountered in high energy heavy ion collisions is such a special case, for the following two reasons:

i) All gluons which undergo scattering are boosted into a part of phase space which was originally empty.

ii) The description simplifies strongly in the rest system of one of the colliding nuclei. For an arbitrary Lorentz frame we do not know how to model the degree of coherence before the collision in both nuclei. In the rest frame of one of the nuclei, however, both nuclei can be approximated by different asymptotic descriptions. For a very fast moving nucleus one observes saturation and can describe the gluon field correlations along the lines of [10, 11], while for the 


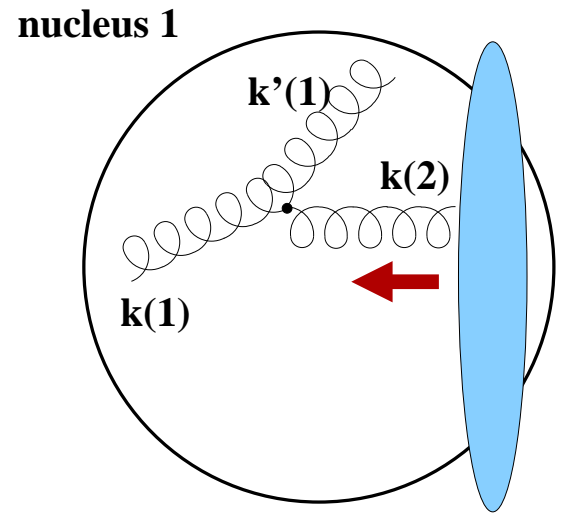

nucleus 2

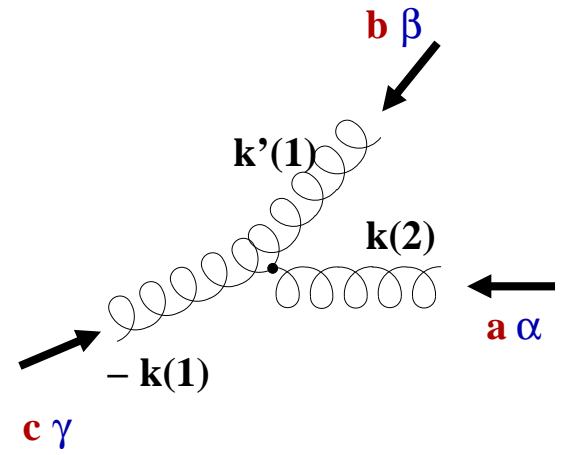

c $\gamma$

FIG. 1: The chosen frame and notation

nucleus at rest we can assume a simple isotropic Gaussian correlation of gluons with a typical virtuality of order $1 / \lambda$. The precise form of this correlation does not really matter, because we will only study the correlations in that part of phase space mentionned in i), which is originally empty.

Therefore, the elementary process of hard gluon scattering is sufficent to estimate the decoherence time.

We perform our calculation in the rest frame of nucleus 1, which is hit by the highly Lorentz contracted nucleus 2 , which is moving to the left. At lowest order in the strong coupling $\alpha_{s}$ the time evolution of the gluon density matrix is governed by the reaction $G_{1}+G_{2} \rightarrow G_{1}^{\prime}$, where $G_{i}$ denotes a component of the gluon wave function of nucleus $i$ (see Figure 11).

We investigate the gluon density matrix $\hat{D}(t)$ in a plane wave basis. Because the ground state wave function of nucleus 1 is a fully coherent bound state, the initial density matrix $\hat{D}(0)$ is strongly non-diagonal in this basis. As usual for a fast moving projectile, it is convenient to use light-cone coordinates $\left(x_{+}, x_{-}, x_{\perp}\right)$ with $\left.x^{ \pm}=\left(x^{0} p m x^{3}\right) / \sqrt{(2}\right)$. Since, in our convention, nucleus 2 moves to the left, $k_{2}^{-}=\left(k^{0}-k^{3}\right) / \sqrt{(2)}=k_{2+}$ is large and the nucleus remains localized in $x^{+}=x_{-}$, which thus denotes the light-cone position of nucleus 2 . On the other hand, $x^{-}=x_{+} \approx \sqrt{2} x^{0}$ takes on the role of the "light-cone time", by which the progress of the collision is monitored. This is also the starting idea of the Color Glass Condensate (CGC) approach [9], which is based on the assumption that most of the gluons in nucleus 2 hardly evolve on the time scale of the collision, endowing the gluon distribution with a "glassy" nature.

The "time" evolution of $\hat{D}$ due to the three-gluon interaction is then given by

$$
\begin{aligned}
\hat{D}\left(d x_{+}\right) & =\hat{U}\left(d x_{+}\right) \hat{D}(0) \hat{U}^{\dagger}\left(d x_{+}\right) \\
& =\hat{D}(0)-\mathrm{i}\left[\hat{H}_{\mathrm{int}}, \hat{D}(0)\right] \frac{d x_{+}}{\sqrt{2}}-\left[\hat{H}_{\mathrm{int}},\left[\hat{H}_{\mathrm{int}}, \hat{D}(0)\right]\right] \frac{\left(d x_{+}\right)^{2}}{4}+\ldots
\end{aligned}
$$

with

$$
\hat{U}\left(d x_{+}\right)=\exp \left(-\mathrm{i} \hat{H}_{\mathrm{int}} d x_{+} / \sqrt{2}\right) .
$$

For what follows, the variable $r$ stands for the multiple quantum numbers completely specifying a gluonic state in nucleus 1: $r=\left\{k, \epsilon^{\beta}, b\right\}$, with wave vector $k$, polarization vector $\epsilon^{\beta}$, and color index $b$. The matrix elements of $H_{\text {int }}$ between two plane wave gluon states of nucleus 1 are then given by

$$
\begin{gathered}
H_{r_{1}, r_{1}^{\prime}} \equiv\left\langle r_{1}\left|H_{\mathrm{int}}\right| r_{1}^{\prime}\right\rangle=\int d^{2} x_{\perp} d x_{-} \int \frac{d k_{2+} d^{2} k_{2 \perp}}{(2 \pi)^{3}} e^{\mathrm{i}\left(k_{2+} x_{-}-k_{2 \perp} \cdot x_{\perp}\right)} e^{\left.\mathrm{i}\left(k_{1+}-k_{1+}^{\prime}\right) x_{-}-\left(k_{1 \perp}-k_{1 \perp}^{\prime}\right) \cdot x_{\perp}\right]} \\
g f_{a b c}\left[g_{\alpha \beta}\left(k_{2}-k_{1}\right)_{\gamma}+g_{\beta \gamma}\left(k_{1}+k_{1}^{\prime}\right)_{\alpha}+g_{\gamma \alpha}\left(-k_{1}^{\prime}-k_{2}\right)_{\beta}\right] \\
{\left[2 k_{1+} k_{1+}^{\prime} V^{2}\right]^{-1 / 2} \epsilon_{1}^{\beta} \epsilon_{1}^{\prime \gamma} 2 \operatorname{Tr}\left[T^{a} A_{\perp}^{\alpha}\left(k_{2 \perp}, k_{2+}\right)\right] .}
\end{gathered}
$$

Here $1 / \sqrt{\sqrt{2} k_{1+} V}$ is the normalization factor for a plane wave gluon in the light-cone formalism. $V$ is some appropriate $(-, \perp)$ normalization volume, which we will specify later. 
$H_{r_{1}, r_{1}^{\prime}}$ describes the interaction with the incident gluons contained in nucleus 2 , which moves very close to the speed of light. Since these gluons originate in many different nucleons, it is a good approximation to consider them as uncorrelated and to describe them as a Gaussian random ensemble 9] representing an incident stream of WeizsäckerWilliams (WW) gluons, all of which have positive $k_{2+}$ momentum.

The gluon fields of nucleus 1 will be characterized by a Gaussian ensemble of off-shell gluon fields with a spatial coherence length $\lambda$ of the order of the confinement scale $\Lambda_{\mathrm{QCD}}^{-1}$ :

$$
\left\langle A_{-}^{a}(x) A_{-}^{b}\left(x^{\prime}\right)\right\rangle=C_{a b} \exp \left\{-\left[\left(x_{\perp}^{\prime}-x_{\perp}\right)^{2}+\left(x_{-}^{\prime}-x_{-}\right)^{2}+\left(x_{+}^{\prime}-x_{+}\right)^{2}\right] / \lambda^{2}\right\} .
$$

We emphasize that we do not assume the gluon field in any nucleus to be dilute. We only assume that the typical momentum transfere $k_{\perp}$ is sufficiently large so that we can treat the interaction perturbatively in the coupling constant $\alpha_{s}=g^{2} / 4 \pi$.

The WW gluon fields are given in 11], eqs. (1-3). For our calculation it is crucially important that the color charge densities $\rho^{a}\left(x_{\perp}, x_{-}\right)$and path ordered factors

$$
S_{0}\left(x_{\perp}, x_{-}\right) \equiv \mathcal{P} \exp \left(i g T^{a} \int d^{2} z_{\perp} \Theta\left(z_{-}-x_{-}\right) \hat{\rho}^{a}\left(z_{\perp}, z_{-}\right) \ln \left(\left|x_{\perp}-z_{\perp}\right| \mu\right)\right)
$$

are uncorrelated for different values of $x_{-}$:

$$
\left\langle\hat{\rho}^{a}\left(x_{\perp}, x_{-}\right) \hat{\rho}^{b}\left(z_{\perp}, z_{-}\right)\right\rangle=\frac{\alpha_{s}}{2 N_{c} \pi} \rho_{N}\left(x_{\perp}, x_{-}\right) \delta\left(x_{-}-z_{-}\right) \delta^{a b} \delta^{2}\left(x_{\perp}-z_{\perp}\right) .
$$

This and eq. (48) of [10] allows to perform the calculation.

Let us stress again that the asymptotic descriptions (4) and eq. (48) of 10] treat the two nuclei in asymmetrically. Therefore, one cannot expect the final expressions to be manifestly boost invariant. Our calculation of the decoherence is specific to our selected Lorentz frame. While a manifestly boost invariant treatment of the decoherence process would be desirable, such a treatment would have to rely on a Lorentz covariant representation of the nuclear ground state. We do not address this interesting problem here.

Because the incident nucleus 2 is a color singlet, its glue field vanishes on average: $\left\langle A_{\perp}^{\alpha}\left(k_{2}\right)\right\rangle=0$. This implies the absence of a contribution to the time evolution of the diagonal elements of the density matrix $\hat{D}$ in first order in $g$. The first nonvanishing term is thus of order $g^{2}$, corresponding to the term proportional to $\left(x_{+}\right)^{2}$ in (1), which is of second order in the WW fields of nucleus 2.

The leading term for the evolution of the density matrix arises from the last term in eq. (10):

$$
D_{r, \hat{r}}(\tau)=D_{r, r}(0)+\sum_{r^{\prime}, \tilde{r}} W_{r, r^{\prime}}(\tau) D_{r^{\prime}, \tilde{r}}(0) W_{\hat{r}, \tilde{r}}^{*}(\tau)
$$

where

$$
W_{r_{1}, r_{1}^{\prime}}(\tau)=\int_{-\tau / 2}^{\tau / 2} d x_{+} H_{r_{1}, r_{1}^{\prime}}\left(x_{+}\right)
$$

Our aim is to calculate the density matrix in the region of phase space populated by the outgoing gluons (momentum $k(1))$. This density matrix contains all the crucial information:

- The ratio

$$
\frac{\operatorname{Tr}\left\{D^{2}\right\}}{(\operatorname{Tr}\{D\})^{2}}
$$

is a measure for the coherence. For a pure state it is one, for a completely decoherent state it is much smaller than one, of the order of $1 / N$ for a region of phase space with $N$ states. We shall show that in the transverse directions (i.e. except for $k_{+}$) we get nearly complete decoherence. We will also see that this ratio depends on the observation time and we shall define as decoherence time $\tau_{\text {deco }}$ as the time after which it reaches $1 / e$.

- The entropy of the final state can be calculated from

$$
S\left(\tau_{f}\right)=\operatorname{Tr}\left\{D\left(\tau_{f}\right) \log D\left(\tau_{f}\right)\right\} .
$$

We argued in [3] that this is a sizeable fraction of the total entropy produced in a heavy ion collision. It should be possible to evaluate Eq.(10) for the density matrix we obtain, but the calculation is highly non-trivial. 
Therefore, we leave this task for a future publication. Having a model for the density matrix in hand it should actually also be possible to calculate other quantities specifying the initial state for the time evolution of the high-temperature phase produced in such collisions.

The symbol $\langle\cdots\rangle$ indicates and average over the WW fields of nucleus 2.

\section{THE DENSITY MATRIX RESULTING FROM HARD GLUONIC INTERACTIONS}

For the WW fields of nucleus 2 we have $k_{2-}=0$, because the fields $A(x)$ do not depend on $x_{+}$. We therefore get

$$
\begin{aligned}
& \left\langle A^{\alpha^{\prime}}\left(k_{2}^{\prime}\right) A^{\tilde{\alpha}}\left(\tilde{k}_{2}\right)\right\rangle=\int d^{4} x^{\prime} d^{4} \tilde{x} e^{-i k_{2}^{\prime} x^{\prime}+i \tilde{k}_{2} \tilde{x}}\left\langle A^{\alpha^{\prime}}\left(x^{\prime}\right) A^{\tilde{\alpha}}(\tilde{x})\right\rangle \\
& =(2 \pi)^{2} \delta\left(k_{2-}^{\prime}\right) \delta\left(\tilde{k}_{2-}\right) \int d^{2} x_{\perp}^{\prime} d^{2} \tilde{x}_{\perp} d x_{-}^{\prime} d \tilde{x}_{-} e^{-\mathrm{i}\left(k_{2+}^{\prime} x_{-}^{\prime}-\tilde{k}_{2+} \tilde{x}_{-}-k_{2 \perp}^{\prime} x_{\perp}^{\prime}+\tilde{k}_{2 \perp} \tilde{x}_{\perp}\right)}\left\langle A^{\alpha^{\prime}}\left(x_{\perp}^{\prime}, x_{-}^{\prime}\right) A^{\tilde{\alpha}}\left(\tilde{x}_{\perp}, \tilde{x}_{-}\right)\right\rangle .
\end{aligned}
$$

The relevant term is now:

$$
\begin{aligned}
\mathcal{W}_{1 \hat{1}, 1^{\prime} \tilde{1}} \equiv & W_{r_{1}, r_{1}^{\prime}} W_{\hat{r}_{1}, \tilde{r}_{1}}^{*} \\
= & \int d k_{2-}^{\prime} \int d \tilde{k}_{2-} \delta\left(k_{2-}^{\prime}+k_{1-}^{\prime}-k_{1-}\right) \delta\left(\tilde{k}_{2-}+\tilde{k}_{1-}-\hat{k}_{1-}\right) \\
& \int d k_{2+}^{\prime} d^{2} k_{2 \perp}^{\prime} \theta\left(k_{2+}^{\prime}\right) \int d \tilde{k}_{2+} d^{2} \tilde{k}_{2 \perp} \theta\left(\tilde{k}_{2+}\right) \delta\left(k_{2+}^{\prime}+k_{1+}^{\prime}-k_{1+}\right) \delta^{2}\left(k_{2 \perp}^{\prime}+k_{1 \perp}^{\prime}-k_{1 \perp}\right) \\
& \delta\left(\tilde{k}_{2+}+\tilde{k}_{1+}-\hat{k}_{1+}\right) \delta^{2}\left(\tilde{k}_{2 \perp}+\tilde{k}_{1 \perp}-\hat{k}_{1 \perp}\right) \\
& g f_{a b c}\left[g_{\alpha \beta}\left(k_{2}^{\prime}-k_{1}^{\prime}\right)_{\gamma}+g_{\beta \gamma}\left(k_{1}^{\prime}+k_{1}\right)_{\alpha}+g_{\gamma \alpha}\left(-k_{1}-k_{2}^{\prime}\right)_{\beta}\right] \\
& g f_{\tilde{a} \tilde{b} c}\left[g_{\tilde{\alpha} \tilde{\beta}}\left(\tilde{k}_{2}-\tilde{k}_{1}\right)_{\tilde{\gamma}}+g_{\tilde{\beta} \tilde{\gamma}}\left(\tilde{k}_{1}+\hat{k}_{1}\right)_{\tilde{\alpha}}+g_{\tilde{\gamma} \tilde{\alpha}}\left(-\hat{k}_{1}-\tilde{k}_{2}\right)\right] \\
& {\left[2 k_{1+} \hat{k}_{1+} V^{2}\right]^{-1 / 2} \epsilon\left(k_{1}^{\prime}\right)^{\beta} \epsilon^{*}\left(\tilde{k}_{1}\right)^{\tilde{\beta}}\left(\sum_{\epsilon} \epsilon\left(k_{1}\right)^{\gamma} \epsilon^{*}\left(\hat{k}_{1}\right)^{\tilde{\gamma}}\right) } \\
& 2 \operatorname{Tr}\left[T^{a} A_{\perp}^{\alpha}\left(k_{2 \perp}^{\prime}, k_{2+}^{\prime}\right)\right] 2 \operatorname{Tr}\left[T^{\tilde{a}} A_{\perp}^{\tilde{\alpha} *}\left(\tilde{k}_{2 \perp}, \tilde{k}_{2+}\right)\right] .
\end{aligned}
$$

Here the first line (the $k_{2-}$ integrals serves more or less only as reminder.) Note that there are no normalization factors for the incomming gluon states, i.e. no factor $\left[2 k_{1+}^{\prime} \tilde{k}_{1+} V^{2}\right]^{-1 / 2}$. The reason is that our initial states are highly virtual, interfering gluon states, i.e. no momentum eigenstates. For the time being we absorb all normalization factors into our definition of $d\left(x, x^{\prime}\right)$ which therefore is not dimensionles but in coordinate space has the dimensions Energy $^{2}$. We do not know how to properly normalize a density matrix for an arbitrary virtual field configuration. Luckily we will only need the normalization of the diagonal elements.

We first focus on the ensemble average of the expression in the last line, involving the WW fields in nucleus 2 :

$$
\begin{aligned}
\langle\mathcal{J}\rangle \equiv & V^{-2}\left\langle 2 \operatorname{Tr}\left[T^{a} A_{\perp}^{\alpha}\left(k_{2 \perp}^{\prime}, k_{2+}^{\prime}\right)\right] 2 \operatorname{Tr}\left[T^{\tilde{a}} A_{\perp}^{\tilde{\alpha} *}\left(\tilde{k}_{2 \perp}, \tilde{k}_{2+}\right)\right]\right\rangle \\
= & V^{-2} \int d^{2} w_{\perp} d w_{-} \delta\left(k_{2-}^{\prime}\right) \delta\left(\tilde{k}_{2-}\right) e^{-\mathrm{i}\left(k_{2+}^{\prime} w_{-}-k_{2 \perp}^{\prime} \cdot w_{\perp}\right)} \int d^{2} \tilde{w}_{\perp} d \tilde{w}_{-} e^{\mathrm{i}\left(\tilde{k}_{2+} \tilde{w}_{-}-\tilde{k}_{2 \perp} \cdot \tilde{w}_{\perp}\right)} \\
& \int d^{2} y_{\perp} d y_{-} \theta\left(y_{-}-w_{-}\right) \int d^{2} \tilde{y}_{\perp} d \tilde{y}_{-} \theta\left(\tilde{y}_{-}-\tilde{w}_{-}\right) \frac{\left(w_{\perp}-y_{\perp}\right)^{\alpha}}{\left|w_{\perp}-\tilde{w}_{\perp}\right|^{2}} \frac{\left(\tilde{y}_{\perp}\right)^{\tilde{\alpha}}}{\left|\tilde{w}_{\perp}-\tilde{y}_{\perp}\right|^{2}} \\
& \left\langle 2 \operatorname{Tr}\left[T^{a} S_{0}\left(w_{\perp}, y_{-}\right) T^{d} S_{0}^{-1}\left(w_{\perp}, y_{-}\right)\right] 2 \operatorname{Tr}\left[T^{\tilde{a}} S_{0}\left(\tilde{w}_{\perp}, \tilde{y}_{-}\right) T^{\tilde{d}} S_{0}^{-1}\left(\tilde{w}_{\perp}, \tilde{y}_{-}\right)\right] \hat{\rho}^{d}\left(y_{\perp}, y_{-}\right) \hat{\rho}^{\tilde{d}}\left(\tilde{y}_{\perp}, \tilde{y}_{-}\right)\right\rangle .
\end{aligned}
$$


We now assume complete factorization of the average, i.e.

$$
\begin{aligned}
& \left\langle 2 \operatorname{Tr}\left[T^{a} S_{0}\left(w_{\perp}, y_{-}\right) T^{d} S_{0}^{-1}\left(w_{\perp}, y_{-}\right)\right] 2 \operatorname{Tr}\left[T^{\tilde{a}} S_{0}\left(\tilde{w}_{\perp}, \tilde{y}_{-}\right) T^{\tilde{d}} S_{0}^{-1}\left(\tilde{w}_{\perp}, \tilde{y}_{-}\right)\right] \hat{\rho}^{d}\left(y_{\perp}, y_{-}\right) \hat{\rho}^{\tilde{d}}\left(\tilde{y}_{\perp}, \tilde{y}_{-}\right)\right\rangle \\
= & \left\langle 2 \operatorname{Tr}\left[T^{a} S_{0}\left(w_{\perp}, y_{-}\right) T^{d} S_{0}^{-1}\left(w_{\perp}, y_{-}\right)\right] 2 \operatorname{Tr}\left[T^{\tilde{a}} S_{0}\left(\tilde{w}_{\perp}, \tilde{y}_{-}\right) T^{\tilde{d}} S_{0}^{-1}\left(\tilde{w}_{\perp}, \tilde{y}_{-}\right)\right]\right\rangle\left\langle\hat{\rho}^{d}\left(y_{\perp}, y_{-}\right) \hat{\rho}^{\tilde{d}}\left(\tilde{y}_{\perp}, \tilde{y}_{-}\right)\right\rangle \\
= & \left\langle 2 \operatorname{Tr}\left[T^{a} S_{0}\left(w_{\perp}, y_{-}\right) T^{d} S_{0}^{-1}\left(w_{\perp}, y_{-}\right)\right] 2 \operatorname{Tr}\left[T^{\tilde{a}} S_{0}\left(\tilde{w}_{\perp}, \tilde{y}_{-}\right) T^{\tilde{d}} S_{0}^{-1}\left(\tilde{w}_{\perp}, \tilde{y}_{-}\right)\right]\right\rangle \\
& \times \frac{\alpha_{s}}{2 \pi N_{c}} \rho_{N}\left(y_{\perp}, y_{-}\right) \delta^{d \tilde{d}} \delta\left(y_{-}-\tilde{y}_{-}\right) \delta^{2}\left(y_{\perp}-\tilde{y}_{\perp}\right) .
\end{aligned}
$$

Using the identity

$$
2 \operatorname{Tr}\left[T^{d} B^{e} T^{e}\right] 2 \operatorname{Tr}\left[T^{d} C^{f} T^{f}\right]=B^{d} C^{d}=2 \operatorname{Tr}\left[B^{e} T^{e} C^{f} T^{f}\right]
$$

to rewrite the product of two color traces as a single trace, we get

$$
\begin{aligned}
\langle\mathcal{J}\rangle= & V^{-2} \int d^{2} w_{\perp} d w_{-} e^{-\mathrm{i}\left(k_{2+}^{\prime} w_{-}-k_{2 \perp}^{\prime} \cdot w_{\perp}\right)} \int d^{2} \tilde{w}_{\perp} d \tilde{w}_{-} e^{\mathrm{i}\left(\tilde{k}_{2+} \tilde{w}_{-}-\tilde{k}_{2 \perp} \cdot \tilde{w}_{\perp}\right)} \delta\left(k_{2-}^{\prime}\right) \delta\left(\tilde{k}_{2-}\right) \\
& \int d^{2} y_{\perp} d y_{-} \theta\left(y_{-}-w_{-}\right) \theta\left(y_{-}-\tilde{w}_{-}\right) \frac{\left(w_{\perp}-y_{\perp}\right)^{\alpha}}{\left|w_{\perp}-y_{\perp}\right|^{2}} \frac{\left(\tilde{w}_{\perp}-y_{\perp}\right)^{\tilde{\alpha}}}{\left|\tilde{w}_{\perp}-y_{\perp}\right|^{2}} \frac{\alpha_{s}}{2 \pi N_{c}} \rho_{N}\left(y_{\perp}, y_{-}\right) \\
& \left\langle 2 \operatorname{Tr}\left[S_{0}^{-1}\left(w_{\perp}, y_{-}\right) T^{a} S_{0}\left(w_{\perp}, y_{-}\right) S_{0}^{-1}\left(\tilde{w}_{\perp}, y_{-}\right) T^{\tilde{a}} S_{0}\left(\tilde{w}_{\perp}, y_{-}\right)\right]\right\rangle .
\end{aligned}
$$

Now we use eq. (47) from [10] and assume that for $a \neq \tilde{a}$ the ensemble average vanishes. The fact that the original expression (47) has $S_{0}$ and $S_{0}^{-1}$ factors interchanged and thus is the complex conjugate of our expression, does not matter, because the result is real.

$$
\begin{aligned}
& \left\langle\operatorname{Tr}\left[S_{0}^{-1}\left(w_{\perp}, y_{-}\right) T^{a} S_{0}\left(w_{\perp}, y_{-}\right) S_{0}^{-1}\left(\tilde{w}_{\perp}, y_{-}\right) T^{\tilde{a}} S_{0}\left(\tilde{w}_{\perp}, y_{-}\right)\right]\right\rangle \\
= & \frac{\delta^{a \tilde{a}}}{N_{c}^{2}-1}\left\langle\operatorname{Tr}\left[S_{0}^{-1}\left(w_{\perp}, y_{-}\right) T^{a} S_{0}\left(w_{\perp}, y_{-}\right) S_{0}^{-1}\left(\tilde{w}_{\perp}, y_{-}\right) T^{a} S_{0}\left(\tilde{w}_{\perp}, y_{-}\right)\right]\right\rangle \\
= & \frac{\delta^{a \tilde{a}} C_{F} N_{c}}{N_{c}^{2}-1} \exp \left(-g^{2} \frac{\pi \rho_{\mathrm{rel}} N_{c}}{4\left(N_{c}^{2}-1\right)} x G\left(x,\left|\tilde{w}_{\perp}-w_{\perp}\right|^{-2}\right)\left(\tilde{w}_{\perp}-w_{\perp}\right)^{2}\left(y_{-}+y_{-}^{(0)}\right)\right)
\end{aligned}
$$

where $y_{-}^{(0)}=\sqrt{R^{2}-x_{\perp}^{2}} / \sqrt{2} \gamma$.

This gives the following result:

$$
\begin{aligned}
\langle\mathcal{J}\rangle= & V^{-2} \int d^{2} w_{\perp} d w_{-} e^{-\mathrm{i}\left(k_{2+}^{\prime} w_{-}-k_{2 \perp}^{\prime} \cdot w_{\perp}\right)} \int d^{2} \tilde{w}_{\perp} d \tilde{w}_{-} e^{\mathrm{i}\left(\tilde{k}_{2+} \tilde{w}_{-}-\tilde{k}_{2 \perp} \cdot \tilde{w}_{\perp}\right)} \delta\left(k_{2-}^{\prime}\right) \delta\left(\tilde{k}_{2-}\right) \\
& \int d^{2} y_{\perp} \int_{-y_{-}^{(0)}}^{y_{-}^{(0)}} d y_{-} \theta\left(y_{-}-w_{-}\right) \theta\left(y_{-}-\tilde{w}_{-}\right) \frac{\left(w_{\perp}-y_{\perp}\right)^{\alpha}}{\left|w_{\perp}-y_{\perp}\right|^{2}} \frac{\left(\tilde{w}_{\perp}-y_{\perp}\right)^{\tilde{\alpha}}}{\left|\tilde{w}_{\perp}-y_{\perp}\right|^{2}} \frac{\alpha_{s}}{2 \pi N_{c}} \rho_{N}\left(y_{\perp}, y_{-}\right) \delta^{a \tilde{a}} \\
& \frac{2 C_{F} N_{c}}{N_{c}^{2}-1} \exp \left(-g^{2} \frac{\pi \rho_{\text {rel }} N_{c}}{4\left(N_{c}^{2}-1\right)} x G\left(x,\left|\tilde{w}_{\perp}-w_{\perp}\right|^{-2}\right)\left(\tilde{w}_{\perp}-w_{\perp}\right)^{2}\left(y_{-}+y_{-}^{(0)}\right)\right)
\end{aligned}
$$

Next we perform the $y_{-}$integration. To do so we make one more approximation. I assume $y_{-}^{(0)} \ll w_{-}, \tilde{w}_{-}$and substitute the $\theta$-functions by $\theta\left(-w_{-}\right) \theta\left(-\tilde{w}_{-}\right)$.

$$
\begin{aligned}
\langle\mathcal{J}\rangle= & V^{-2} \int d^{2} w_{\perp} \int_{-\infty}^{0} d w_{-} e^{-\mathrm{i}\left(k_{2+}^{\prime} w_{-}-k_{2 \perp}^{\prime} \cdot w_{\perp}\right)} \int d^{2} \tilde{w}_{\perp} \int_{-\infty}^{0} d \tilde{w}_{-} e^{\mathrm{i}\left(\tilde{k}_{2+} \tilde{w}_{-}-\tilde{k}_{2 \perp} \cdot \tilde{w}_{\perp}\right)} \delta\left(k_{2-}^{\prime}\right) \delta\left(\tilde{k}_{2-}\right) \\
& \int d^{2} y_{\perp} \delta^{a \tilde{a}} \frac{\left(w_{\perp}-y_{\perp}\right)^{\alpha}}{\left|w_{\perp}-y_{\perp}\right|^{2}} \frac{\left(\tilde{w}_{\perp}-y_{\perp}\right)^{\tilde{\alpha}}}{\left|\tilde{w}_{\perp}-y_{\perp}\right|^{2}} \frac{4\left(N_{c}^{2}-1\right) 2 y_{-}^{(0)}}{g^{2} \pi N_{c} \rho_{\mathrm{rel}}\left|\tilde{w}_{\perp}-w_{\perp}\right|^{2} x G\left(x,\left|\tilde{w}_{\perp}-w_{\perp}\right|^{-2}\right)} \\
& {\left[1-\exp \left(-g^{2} \frac{2 y_{-}^{(0)} \pi \rho_{\mathrm{rel}} N_{c}}{4\left(N_{c}^{2}-1\right)}\left(\tilde{w}_{\perp}-w_{\perp}\right)^{2} x G\left(x,\left|\tilde{w}_{\perp}-w_{\perp}\right|^{-2}\right)\right)\right] \frac{\alpha_{s}}{2 \pi N_{c}} \rho_{N}\left(y_{\perp}, y_{-}\right) }
\end{aligned}
$$


In the spirit of the discussion above we assume

$$
\frac{\rho_{N}\left(y_{\perp}, y_{-}\right)}{2 y_{-}^{(0)} \rho_{\text {rel }}}=1
$$

and use the relation (valid at leading logarithmic accuracy)

$$
\frac{\partial x G\left(x, Q^{2}\right)}{\partial \ln Q^{2}}=\frac{\alpha_{s}\left(N_{c}^{2}-1\right)}{2 \pi N_{c}} \quad \longrightarrow \quad x G\left(x, Q^{2}\right)=\frac{\alpha_{s}\left(N_{c}^{2}-1\right)}{2 \pi N_{c}} \ln \left(Q^{2} / \mu^{2}\right)
$$

to obtain

$$
\begin{aligned}
\langle\mathcal{J}\rangle= & V^{-2} \int d^{2} w_{\perp} \int_{-\infty}^{0} d w_{-} e^{-\mathrm{i}\left(k_{2+}^{\prime} w_{-}-k_{2 \perp}^{\prime} \cdot w_{\perp}\right)} \int d^{2} \tilde{w}_{\perp} \int_{-\infty}^{0} d \tilde{w}_{-} e^{\mathrm{i}\left(\tilde{k}_{2+} \tilde{w}_{-}-\tilde{k}_{2 \perp} \cdot \tilde{w}_{\perp}\right)} \\
& \int d^{2} y_{\perp} \frac{\left(w_{\perp}-y_{\perp}\right)^{\alpha}}{\left|w_{\perp}-y_{\perp}\right|^{2}} \frac{\left(\tilde{w}_{\perp}-y_{\perp}\right)^{\tilde{\alpha}}}{\left|\tilde{w}_{\perp}-y_{\perp}\right|^{2}} \delta^{a \tilde{a}} \frac{-4}{g^{2} \pi N_{c}\left(\tilde{w}_{\perp}-w_{\perp}\right)^{2} \ln \left(\mu^{2}\left|\tilde{w}_{\perp}-w_{\perp}\right|^{2}\right)} \\
& {\left[1-\exp \left(-g^{2} \frac{2 y_{-}^{(0)} \pi \rho_{\text {rel }} N_{c}}{4\left(N_{c}^{2}-1\right)}\left(\tilde{w}_{\perp}-w_{\perp}\right)^{2} x G\left(x,\left|\tilde{w}_{\perp}-w_{\perp}\right|^{-2}\right)\right)\right] \delta\left(k_{2-}^{\prime}\right) \delta\left(\tilde{k}_{2-}\right) }
\end{aligned}
$$

Next, using the two dimensional Green's function

$$
\frac{\left(w_{\perp}-y_{\perp}\right)^{\alpha}}{\left|w_{\perp}-y_{\perp}\right|^{2}}=-\mathrm{i} \int \frac{d^{2} q_{\perp}}{2 \pi} e^{\mathrm{i} q_{\perp} \cdot\left(w_{\perp}-y_{\perp}\right)} \frac{q_{\perp}^{\alpha}}{q_{\perp}^{2}}
$$

the $y_{\perp}$ integral can be performed:

$$
\begin{aligned}
& \int d^{2} y_{\perp} \frac{\left(w_{\perp}-y_{\perp}\right)^{\alpha}}{\left|w_{\perp}-y_{\perp}\right|^{2}} \frac{\left(\tilde{w}_{\perp}-y_{\perp}\right)^{\tilde{\alpha}}}{\left|\tilde{w}_{\perp}-y_{\perp}\right|^{2}}=-\int d^{2} y_{\perp} \int \frac{d^{2} q_{\perp}}{2 \pi} \frac{d^{2} \tilde{q}_{\perp}}{2 \pi} e^{\mathrm{i}\left[q_{\perp} \cdot\left(w_{\perp}-y_{\perp}\right)+\tilde{q}_{\perp} \cdot\left(\tilde{w}_{\perp}-y_{\perp}\right)\right]} \frac{q_{\perp}^{\alpha}}{q_{\perp}^{2}} \frac{\tilde{q}_{\perp}^{\alpha}}{\tilde{q}_{\perp}^{2}} \\
& =\int d^{2} q_{\perp} e^{\mathrm{i} q_{\perp} \cdot\left(w_{\perp}-\tilde{w}_{\perp}\right)} \frac{q_{\perp}^{\alpha} q_{\perp}^{\tilde{\alpha}}}{\left(q_{\perp}^{2}\right)^{2}} \\
& =\delta^{\alpha \tilde{\alpha}} \int_{0}^{\infty} \frac{d q_{\perp}}{q_{\perp}} \int_{0}^{2 \pi} d \phi e^{\mathrm{i} q_{\perp}\left|w_{\perp}-\tilde{w}_{\perp}\right| \cos \phi}\left(\begin{array}{c}
\cos ^{2} \phi \\
\sin ^{2} \phi
\end{array}\right) \\
& =2 \pi \delta^{\alpha \tilde{\alpha}} \int_{0}^{\infty} \frac{d q_{\perp}}{q_{\perp}},\left(\begin{array}{c}
J_{1}^{\prime}\left(q_{\perp}\left|w_{\perp}-\tilde{w}_{\perp}\right|\right) \\
J_{1}^{\prime}\left(q_{\perp}\left|w_{\perp}-\tilde{w}_{\perp}\right|\right)+J_{2}\left(q_{\perp}\left|w_{\perp}-\tilde{w}_{\perp}\right|\right)
\end{array}\right)
\end{aligned}
$$

Up to the logarithmic divergence at $q_{\perp} \rightarrow 0$ this integral is independent of $\left|w_{\perp}-\tilde{w}_{\perp}\right|$ as one can see by substituting $q_{\perp}\left|w_{\perp}-\tilde{w}_{\perp}\right| \rightarrow z$. To regularize the IR divergence we introduce a lower integration boundary for $z$ in the form $\mu\left|w_{\perp}-\tilde{w}_{\perp}\right|$ and insert the finite $z \rightarrow 0$ limit of the Bessel functions: $J_{0}^{\prime}(0)=1, J_{2}(-)=0$, obtaining:

$$
\delta^{\alpha \tilde{\alpha}} \int_{\mu\left|w_{\perp}-\tilde{w}_{\perp}\right|}^{\infty} \frac{d z}{z}=-\frac{1}{2} \delta^{\alpha \tilde{\alpha}} \ln \left(\mu^{2}\left|w_{\perp}-\tilde{w}_{\perp}\right|^{2}\right) .
$$

We thus finally end up with the result

$$
\begin{aligned}
\langle\mathcal{J}\rangle= & V^{-2} \int d^{2} w_{\perp} \int_{-\infty}^{0} d w_{-} e^{-\mathrm{i}\left(k_{2+}^{\prime} w_{-}-k_{2 \perp}^{\prime} \cdot w_{\perp}\right)} \int d^{2} \tilde{w}_{\perp} \int_{-\infty}^{0} d \tilde{w}_{-} e^{\mathrm{i}\left(\tilde{k}_{2+} \tilde{w}_{-}-\tilde{k}_{2 \perp} \cdot \tilde{w}_{\perp}\right)} \delta^{a \tilde{a}} \delta^{\alpha \tilde{\alpha}} \frac{2}{g^{2} N_{c}\left|\tilde{w}_{\perp}-w_{\perp}\right|^{2}} \\
& {\left[1-\exp \left(-g^{2} \frac{2 y_{-}^{(0)} \pi \rho_{\mathrm{rel}} N_{c}}{4\left(N_{c}^{2}-1\right)}\left(\tilde{w}_{\perp}-w_{\perp}\right)^{2} x G\left(x,\left|\tilde{w}_{\perp}-w_{\perp}\right|^{-2}\right)\right)\right] \delta\left(k_{2-}^{\prime}\right) \delta\left(\tilde{k}_{2-}\right) }
\end{aligned}
$$

I insert a suitable $\epsilon$-prescription to perform the integration over $w_{-}$:

$$
\begin{aligned}
\langle\mathcal{J}\rangle= & \frac{2 \delta^{a \tilde{a}} \delta^{\alpha \tilde{\alpha}}}{g^{2} N_{c} V^{2}} \int \frac{d^{2} w_{\perp} d^{2} \tilde{w}_{\perp}}{\left|\tilde{w}_{\perp}-w_{\perp}\right|^{2}} e^{\mathrm{i}\left(k_{2 \perp} \cdot w_{\perp}-\tilde{k}_{2 \perp} \cdot \tilde{w}_{\perp}\right)} \int_{-\infty}^{0} d w_{-} \int_{-\infty}^{0} d \tilde{w}_{-} e^{-\mathrm{i}\left(\left(k_{2+}+\mathrm{i} \epsilon\right) w_{-}-\left(\tilde{k}_{2+}-\mathrm{i} \epsilon\right) \tilde{w}_{-}\right)} \\
& {\left[1-\exp \left(-g^{2} \frac{2 y_{-}^{(0)} \pi \rho_{\mathrm{rel}} N_{c}}{4\left(N_{c}^{2}-1\right)}\left(\tilde{w}_{\perp}-w_{\perp}\right)^{2} x G\left(x,\left|\tilde{w}_{\perp}-w_{\perp}\right|^{-2}\right)\right)\right] \delta\left(k_{2-}^{\prime}\right) \delta\left(\tilde{k}_{2-}\right) } \\
= & \frac{2 \delta^{a \tilde{a}} \delta^{\alpha \tilde{\alpha}}}{g^{2} N_{c} V^{2}} \int d^{2} w_{\perp} \frac{\mathrm{i}}{k_{2+}^{\prime}+\mathrm{i} \epsilon} e^{\mathrm{i} k_{2 \perp} \cdot w_{\perp}} \int d^{2} \tilde{w}_{\perp} \frac{-\mathrm{i}}{\tilde{k}_{2+}-\mathrm{i} \epsilon} e^{-\mathrm{i} \tilde{k}_{2 \perp} \cdot \tilde{w}_{\perp}}\left|\tilde{w}_{\perp}-w_{\perp}\right|^{-2} \\
& {\left[1-\exp \left(-g^{2} \frac{2 y_{-}^{(0)} \pi \rho_{\mathrm{rel}} N_{c}}{4\left(N_{c}^{2}-1\right)}\left(\tilde{w}_{\perp}-w_{\perp}\right)^{2} x G\left(x,\left|\tilde{w}_{\perp}-w_{\perp}\right|^{-2}\right)\right)\right] \delta\left(k_{2-}^{\prime}\right) \delta\left(\tilde{k}_{2-}\right) }
\end{aligned}
$$


Next we substitute $Z_{\perp}=w_{\perp}+\tilde{w}_{\perp}$ and $z_{\perp}=w_{\perp}-\tilde{w}_{\perp}$, giving

$$
\begin{aligned}
\langle\mathcal{J}\rangle= & \frac{2 \delta^{a \tilde{a}} \delta^{\alpha \tilde{\alpha}}}{g^{2} N_{c} V^{2}} \frac{1}{\left(k_{2+}^{\prime}+\mathrm{i} \epsilon\right)\left(\tilde{k}_{2+}-\mathrm{i} \epsilon\right)} \int d^{2} Z_{\perp} \int d^{2} z_{\perp} e^{\mathrm{i}\left[k_{2 \perp}^{\prime} \cdot\left(Z_{\perp}+z_{\perp}\right) / 2-\tilde{k}_{2 \perp} \cdot\left(Z_{\perp}-z_{\perp}\right) / 2\right]} \\
& {\left[1-\exp \left(-g^{2} \frac{2 y_{-}^{(0)} \pi \rho_{\mathrm{rel}} N_{c} z_{\perp}^{2}}{4\left(N_{c}^{2}-1\right)} x G\left(x,\left|z_{\perp}\right|^{-2}\right)\right)\right] \delta\left(k_{2-}^{\prime}\right) \delta\left(\tilde{k}_{2-}\right) }
\end{aligned}
$$

Now we can insert the definition of the saturation scale from eq. (17) of [1]. In doing so we identify $r$ in that equation with $y_{-}^{(0)}$ :

$$
g^{2} \frac{2 y_{-}^{(0)} \pi \rho_{\mathrm{rel}} N_{c} z_{\perp}^{2}}{4\left(N_{c}^{2}-1\right)} x G\left(x,\left|z_{\perp}\right|^{-2}\right)=\frac{Q_{s}^{2} z_{\perp}^{2}}{4}
$$

This gives:

$$
\langle\mathcal{J}\rangle=\frac{2 \delta^{a \tilde{a}} \delta^{\alpha \tilde{\alpha}}(2 \pi)^{2}}{g^{2} N_{c} V^{2}} \frac{\delta^{2}\left(k_{2 \perp}^{\prime}-\tilde{k}_{2 \perp}\right)}{\left(k_{2+}^{\prime}+\mathrm{i} \epsilon\right)\left(\tilde{k}_{2+}-\mathrm{i} \epsilon\right)} \int \frac{d^{2} z_{\perp}}{z_{\perp}^{2}} e^{\mathrm{i} k_{2 \perp}^{\prime} \cdot z_{\perp}}\left[1-\exp \left(-\frac{Q_{s}^{2} z_{\perp}^{2}}{4}\right)\right] \delta\left(k_{2-}^{\prime}\right) \delta\left(\tilde{k}_{2-}\right)
$$

To do the $z_{\perp}$-integral we use the fact that the integral is well behaved at $z_{\perp} \rightarrow 0$ and we assume some $\epsilon$ prescription to make it convergent at $z_{\perp} \rightarrow \infty$.

$$
K \equiv \int \frac{d^{2} z_{\perp}}{z_{\perp}^{2}} e^{\mathrm{i} k_{2 \perp}^{\prime} \cdot z_{\perp}}\left[1-\exp \left(-\frac{Q_{s}^{2} z_{\perp}^{2}}{4}\right)\right]=\frac{Q_{s}^{2}}{4} \int_{0}^{1} d u \int d^{2} z_{\perp} e^{\mathrm{i} k_{2 \perp}^{\prime} \cdot z_{\perp}} \exp \left(-\frac{Q_{s}^{2} z_{\perp}^{2} u}{4}\right)
$$

Substituting $z_{\perp} \rightarrow z_{\perp}+\mathrm{i} 2 k_{2 \perp}^{\prime} /\left(Q_{s}^{2} u\right)$, we get:

$$
K=\frac{Q_{s}^{2}}{4} \int_{0}^{1} d u \int d^{2} z_{\perp} \exp \left(-\frac{Q_{s}^{2} z_{\perp}^{2} u}{4}-\frac{{k_{2 \perp}^{\prime}}_{2}}{Q_{s}^{2} u}\right)=\pi \int_{0}^{1} \frac{d u}{u} \exp \left(-\frac{k_{2 \perp}^{\prime 2}}{Q_{s}^{2} u}\right)
$$

Next we substitute $u \rightarrow{k^{\prime}}_{2 \perp}^{2} /\left(Q_{s}^{2} t\right)$ to obtain:

$$
K=\pi \int_{k_{2 \perp}^{\prime 2} / Q_{s}^{2}}^{\infty} \frac{d t}{t} e^{-t}=\pi \mathrm{E}_{1}\left(\frac{k_{2 \perp}^{\prime 2}}{Q_{s}^{2}}\right)
$$

yielding finally

$$
\langle\mathcal{J}\rangle=\frac{\delta^{a \tilde{a}} \delta^{\alpha \tilde{\alpha}}(2 \pi)^{3}}{g^{2} N_{c} V^{2}} \frac{\delta^{2}\left(k_{2 \perp}^{\prime}-\tilde{k}_{2 \perp}\right)}{\left(k_{2+}^{\prime}+\mathrm{i} \epsilon\right)\left(\tilde{k}_{2+}-\mathrm{i} \epsilon\right)} \mathrm{E}_{1}\left(\frac{{k_{2 \perp}^{\prime}}^{2}}{Q_{s}^{2}}\right) \delta\left(k_{2-}^{\prime}\right) \delta\left(\tilde{k}_{2-}\right)
$$


thus we get:

$$
\begin{aligned}
& \mathcal{W}_{1 \hat{1}, 1^{\prime} \tilde{1}}=\int d k_{2-}^{\prime} \int d \tilde{k}_{2-} \delta\left(k_{2-}^{\prime}+k_{1-}^{\prime}-k_{1-}\right) \delta\left(\tilde{k}_{2-}+\tilde{k}_{1-}-\hat{k}_{1-}\right) \delta\left(k_{2-}^{\prime}\right) \delta\left(\tilde{k}_{2-}\right) \\
& \frac{(2 \pi)^{3}}{V} f_{a b c} f_{a \tilde{b} \hat{c}} \int d k_{2+}^{\prime} d^{2} k_{2 \perp}^{\prime} \theta\left(k_{2+}^{\prime}\right) \frac{1}{\sqrt{2 k_{1+} \hat{k}_{1+}}} \int d \tilde{k}_{2+} d^{2} \tilde{k}_{2 \perp} \theta\left(\tilde{k}_{2+}\right) \delta\left(k_{2+}^{\prime}+k_{1+}^{\prime}-k_{1+}\right) \\
& \delta^{2}\left(k_{2 \perp}^{\prime}+k_{1 \perp}^{\prime}-k_{1 \perp}\right) \delta\left(\tilde{k}_{2+}+\tilde{k}_{1+}-\hat{k}_{1+}\right) \delta^{2}\left(\tilde{k}_{2 \perp}+\tilde{k}_{1 \perp}-\hat{k}_{1 \perp}\right) \delta^{2}\left(k_{2 \perp}^{\prime}-\tilde{k}_{2 \perp}\right) \\
& {\left[g_{\alpha \beta}\left(k_{2}^{\prime}-k_{1}^{\prime}\right)_{\gamma}+g_{\beta \gamma}\left(k_{1}^{\prime}+k_{1}\right)_{\alpha}+g_{\gamma \alpha}\left(-k_{1}-k_{2}^{\prime}\right)_{\beta}\right]} \\
& {\left.\left[g_{\tilde{\beta}}^{\alpha}\left(\tilde{k}_{2}-\tilde{k}_{1}\right)_{\tilde{\gamma}}+g_{\tilde{\beta} \tilde{\gamma}}\left(\tilde{k}_{1}+\hat{k}_{1}\right)^{\alpha}+g_{\tilde{\gamma}}^{\alpha}\left(-\hat{k}_{1}-\tilde{k}_{2}\right)_{\tilde{\beta}}\right]\right|_{\alpha=1,2}} \\
& \frac{1}{\left(k_{2+}^{\prime}+\mathrm{i} \epsilon\right)\left(\tilde{k}_{2+}-\mathrm{i} \epsilon\right)} \mathrm{E}_{1}\left(\frac{k_{2 \perp}^{\prime 2}}{Q_{s}^{2}}\right) \epsilon\left(k_{1}^{\prime}\right)^{\beta} \epsilon^{*}\left(\tilde{k}_{1}\right)^{\tilde{\beta}}\left(\sum_{\epsilon} \epsilon\left(k_{1}\right)^{\gamma} \epsilon^{*}\left(\hat{k}_{1}\right)^{\tilde{\gamma}}\right)
\end{aligned}
$$

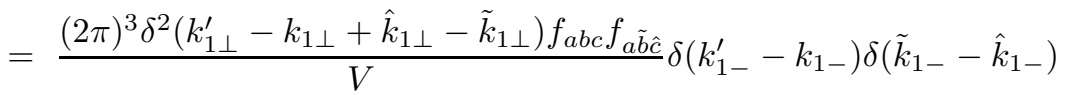

$$
\begin{aligned}
& \int d k_{2+}^{\prime} \theta\left(k_{2+}^{\prime}\right) \int d \tilde{k}_{2+} \theta\left(\tilde{k}_{2+}\right) \frac{1}{\sqrt{2 k_{1+} \hat{k}_{1+}}} \delta\left(k_{2+}^{\prime}+k_{1+}^{\prime}-k_{1+}\right) \delta\left(\tilde{k}_{2+}+\tilde{k}_{1+}-\hat{k}_{1+}\right) \\
& {\left[g_{\alpha \beta}\left(k_{2}^{\prime}-k_{1}^{\prime}\right)_{\gamma}+g_{\beta \gamma}\left(k_{1}^{\prime}+k_{1}\right)_{\alpha}+g_{\gamma \alpha}\left(-k_{1}-k_{2}^{\prime}\right)_{\beta}\right]} \\
& {\left.\left[g_{\tilde{\beta}}^{\alpha}\left(\tilde{k}_{2}-\tilde{k}_{1}\right)_{\tilde{\gamma}}+g_{\tilde{\beta} \tilde{\gamma}}\left(\tilde{k}_{1}+\hat{k}_{1}\right)^{\alpha}+g_{\tilde{\gamma}}^{\alpha}\left(-\hat{k}_{1}-\tilde{k}_{2}\right)_{\tilde{\beta}}\right]\right|_{\alpha=1,2}} \\
& \frac{1}{\left(k_{2+}^{\prime}+\mathrm{i} \epsilon\right)\left(\tilde{k}_{2+}-\mathrm{i} \epsilon\right)} \mathrm{E}_{1}\left(\frac{k_{2 \perp}^{\prime 2}}{Q_{s}^{2}}\right) \epsilon\left(k_{1}^{\prime}\right)^{\beta} \epsilon^{*}\left(\tilde{k}_{1}\right)^{\tilde{\beta}}\left(\sum_{\epsilon} \epsilon\left(k_{1}\right)^{\gamma} \epsilon^{*}\left(\hat{k}_{1}\right)^{\tilde{\gamma}}\right) \\
& =\frac{(2 \pi)^{3} \delta^{2}\left(k_{1 \perp}^{\prime}-k_{1 \perp}+\hat{k}_{1 \perp}-\tilde{k}_{1 \perp}\right) f_{a b c} f_{a \tilde{b} \hat{c}}}{V \sqrt{2 k_{1+} \hat{k}_{1+}}} \theta\left(\hat{k}_{1+}-\tilde{k}_{1+}\right) \theta\left(k_{1+}-k_{1+}^{\prime}\right) \delta\left(k_{1-}^{\prime}-k_{1-}\right) \delta\left(\tilde{k}_{1-}-\hat{k}_{1-}\right) \\
& {\left[g_{\alpha \beta}\left(k_{2}^{\prime}-k_{1}^{\prime}\right)_{\gamma}+g_{\beta \gamma}\left(k_{1}^{\prime}+k_{1}\right)_{\alpha}+g_{\gamma \alpha}\left(-k_{1}-k_{2}^{\prime}\right)_{\beta}\right]} \\
& {\left.\left[g_{\tilde{\beta}}^{\alpha}\left(\tilde{k}_{2}-\tilde{k}_{1}\right)_{\tilde{\gamma}}+g_{\tilde{\beta} \tilde{\gamma}}\left(\tilde{k}_{1}+\hat{k}_{1}\right)^{\alpha}+g_{\tilde{\gamma}}^{\alpha}\left(-\hat{k}_{1}-\tilde{k}_{2}\right)_{\tilde{\beta}}\right]\right|_{\alpha=1,2}} \\
& \frac{1}{\left(k_{1+}-k_{1+}^{\prime}+\mathrm{i} \epsilon\right)\left(\hat{k}_{1+}-\tilde{k}_{1+}-\mathrm{i} \epsilon\right)} \mathrm{E}_{1}\left(\frac{{k_{2 \perp}^{\prime}}_{2 \perp}^{2}}{Q_{s}^{2}}\right) \epsilon\left(k_{1}^{\prime}\right)^{\beta} \epsilon^{*}\left(\tilde{k}_{1}\right)^{\tilde{\beta}}\left(\sum_{\epsilon} \epsilon\left(k_{1}\right)^{\gamma} \epsilon^{*}\left(\hat{k}_{1}\right)^{\tilde{\gamma}}\right)
\end{aligned}
$$

with $k_{2 \perp}^{\prime}=k_{1 \perp}-k_{1 \perp}^{\prime}$ and $\tilde{k}_{2 \perp}=\hat{k}_{1 \perp}-\tilde{k}_{1 \perp}$.

The WW-gluons are on-shell, i.e. $k_{2-}^{\prime}=\tilde{k}_{2-}=0$ and thus $k_{1-}=k_{1-}^{\prime}$ and $\hat{k}_{1-}=\tilde{k}_{1-}$. Therefore we can substitute in general $k_{2}^{\prime}=k_{1}-k_{1}^{\prime}$ and $\tilde{k}_{2}=\hat{k}_{1}-\tilde{k}_{1}$.

$$
\begin{aligned}
\mathcal{W}_{1 \hat{1}, 1^{\prime} \tilde{1}}= & \frac{(2 \pi)^{3} \delta^{2}\left(k_{1 \perp}^{\prime}-k_{1 \perp}+\hat{k}_{1 \perp}-\tilde{k}_{1 \perp}\right) f_{a b c} f_{a \tilde{b} \hat{c}}}{V \sqrt{2 k_{1+} \hat{k}_{1+}}} \theta\left(\hat{k}_{1+}-\tilde{k}_{1+}\right) \theta\left(k_{1+}-k_{1+}^{\prime}\right) \delta\left(k_{1-}^{\prime}-k_{1-}\right) \delta\left(\tilde{k}_{1-}-\hat{k}_{1-}\right) \\
& {\left[g_{\alpha \beta}\left(k_{1}-2 k_{1}^{\prime}\right)_{\gamma}+g_{\beta \gamma}\left(k_{1}^{\prime}+k_{1}\right)_{\alpha}+g_{\gamma \alpha}\left(-2 k_{1}+k_{1}^{\prime}\right)_{\beta}\right] } \\
& {\left.\left[g_{\alpha}^{\tilde{\beta}}\left(\hat{k}_{1}-2 \tilde{k}_{1}\right)_{\tilde{\gamma}}+g_{\tilde{\beta} \tilde{\gamma}}\left(\tilde{k}_{1}+\hat{k}_{1}\right)^{\alpha}+g_{\tilde{\gamma}}^{\alpha}\left(-2 \hat{k}_{1}+\tilde{k}_{1}\right)_{\tilde{\beta}}\right]\right|_{\alpha=1,2} } \\
& \frac{1}{\left(k_{1+}-k_{1+}^{\prime}+\mathrm{i} \epsilon\right)\left(\hat{k}_{1+}-\tilde{k}_{1+}-\mathrm{i} \epsilon\right)} \mathrm{E}_{1}\left(\frac{k_{2 \perp}^{\prime 2}}{Q_{s}^{2}}\right) \epsilon\left(k_{1}^{\prime}\right)^{\beta} \epsilon^{*}\left(\tilde{k}_{1}\right)^{\tilde{\beta}}\left(\sum_{\epsilon} \epsilon\left(k_{1}\right)^{\gamma} \epsilon^{*}\left(\hat{k}_{1}\right)^{\tilde{\gamma}}\right)
\end{aligned}
$$

At this point we have to make an assumption about how far in rapidity a gluon of nucleus 1 is scattered. Let us require an average rapidity $Y>1$ of the scattered gluons (see Fig. 21). Decoherence will be effective, when $Y$ is large 


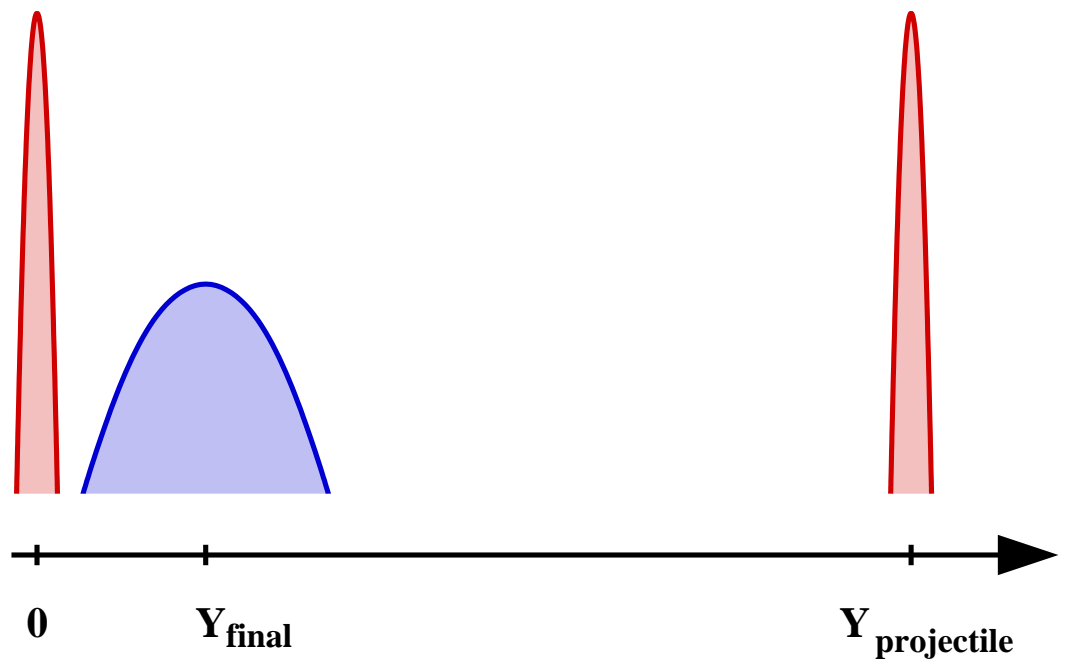

FIG. 2: The rapidity regions considered

enough that the final phase space is originally empty or only sparsely occupied, which is the case for $Y>1$. This condition also implies $\hat{k}_{1+}, k_{1+} \gg k_{1+}^{\prime}, \tilde{k}_{1+}$. Decoherence may also occur inside the target region $(Y \leq 1)$, but we are not concerned with this question here.

With these assumptions it is now possible to greatly simplify Eq. (36). In the square brackets we keep only $k_{1,0}$, $\hat{k}_{1,0}$ and $k_{1,3}, \hat{k}_{1,3}$. We neglect $k_{1, \alpha}$, because $\alpha$ denotes a transverse direction. We can also drop $k_{1, \gamma}$ and $k_{1, \tilde{\gamma}}$ because of the projector at the end of Eq. (36). Thus we are left with

$$
\begin{aligned}
\left.2 g_{\gamma \alpha} k_{1, \beta} g_{\tilde{\gamma}}^{\alpha} 2 \hat{k}_{1, \tilde{\beta}} \epsilon\left(k_{1}^{\prime}\right)^{\beta} \epsilon^{*}\left(\tilde{k}_{1}\right)^{\tilde{\beta}}\left(g^{\gamma \tilde{\gamma}}-\frac{k_{1}^{\gamma} \hat{k}_{1}^{\tilde{\gamma}}}{k_{1} \cdot \hat{k}_{1}}\right)\right|_{\alpha=1,2} & =8 k_{1} \cdot \epsilon\left(k_{1}^{\prime}\right) \hat{k}_{1} \cdot \epsilon^{*}\left(\tilde{k}_{1}\right)\left(1+\frac{k_{1, \perp} \cdot \hat{k}_{1, \perp}}{2 k_{1} \cdot \hat{k}_{1}}\right) \\
& =8 k_{1+} \hat{k}_{1+} \epsilon\left(k_{1}^{\prime}\right)_{-} \epsilon^{*}\left(\tilde{k}_{1}\right)_{-}\left(1+\frac{k_{1, \perp} \cdot \hat{k}_{1, \perp}}{2 k_{1} \cdot \hat{k}_{1}}\right)
\end{aligned}
$$

Making use of:

$$
\sum_{\epsilon} \epsilon\left(k_{1}\right)^{\gamma} \epsilon^{*}\left(\hat{k}_{1}\right)^{\tilde{\gamma}}=\left(g^{\gamma \tilde{\gamma}}-\frac{k_{1}^{\gamma} \hat{k}_{1}^{\tilde{\gamma}}}{k_{1} \cdot \hat{k}_{1}}\right)
$$

The next task is to evaluate the correlator

$$
\left\langle\epsilon\left(k_{1}^{\prime}\right)_{-} \epsilon^{*}\left(\tilde{k}_{1}\right)_{-}\right\rangle
$$

Next we have to evaluate the convolution with the density matrix of the product of polarization vectors and the color factor:

$$
\left\langle f_{a b c} f_{a \tilde{b} \hat{c}} \epsilon\left(k_{1}^{\prime}\right)_{-} \epsilon^{*}\left(\tilde{k}_{1}\right)_{-}\right\rangle_{\text {polarization,color }} \sim \sum_{\epsilon} \sum_{b, \tilde{b}} \epsilon\left(k_{1}^{\prime}\right)_{-} \epsilon^{*}\left(\tilde{k}_{1}\right)_{-} f_{a b c} f_{a \tilde{b} \hat{c}} D\left(b, \tilde{b}, \epsilon\left(k_{1}^{\prime}\right), \epsilon\left(\tilde{k}_{1}^{\prime}\right) ; k_{1}^{\prime}, \tilde{k}_{1}\right)
$$

To do so we Fourier transform into coordinate space and simply assume that there (in the rest frame of nucleus 1) the correlation of the polarization vectors and colors is given by a simple Gaussian with correlation length $\lambda$, i.e. we 
assume that $D$ is a Gaussian in coordinate space.

$$
\begin{aligned}
\left\langle f_{a b c} f_{a \tilde{b} \hat{c}} \epsilon\left(k_{1}^{\prime}\right)_{-} \epsilon^{*}\left(\tilde{k}_{1}\right)_{-}\right\rangle= & C \delta_{c \hat{c}} \int d^{2} y_{\perp}^{\prime} d^{2} \tilde{y}_{\perp} d y_{-}^{\prime} d \tilde{y}_{-} d y_{+}^{\prime} d \tilde{y}_{+} \\
& \left.\exp \left\{\mathrm{i}\left(k_{1, \perp}^{\prime} \cdot y_{\perp}^{\prime}-\tilde{k}_{1, \perp} \cdot \tilde{y}_{\perp}-k_{1,+}^{\prime} y_{-}^{\prime}+\tilde{k}_{+} \tilde{y}_{-}\right)-k_{1,-}^{\prime} y_{+}^{\prime}+\tilde{k}_{-} \tilde{y}_{+}\right)\right\} \\
& \exp \left\{-\left[\left(y_{\perp}^{\prime}-\tilde{y}_{\perp}\right)^{2}+\left(y_{-}^{\prime}-\tilde{y}_{-}\right)^{2}+\left(y_{+}^{\prime}-\tilde{y}_{+}\right)^{2}\right] / \lambda^{2}\right\} \\
= & \frac{C \delta_{c \hat{c}}}{16} \int d^{4} Y^{(+)} d^{4} Y^{(-)}-\exp \left\{-\frac{\mathrm{i}}{2}\left[\left(k_{1}^{\prime}-\tilde{k}_{1}\right) \cdot Y^{(+)}+\left(k_{1}^{\prime}+\tilde{k}_{1}\right) \cdot Y^{(-)}\right]\right\} \\
& \exp \left\{\left[\left(Y_{+}^{(-)}\right)^{2}+\left(Y_{-}^{(-)}\right)^{2}+\left(Y_{\perp}^{(-)}\right)^{2}\right] / \lambda^{2}\right\} \\
= & C \delta_{c \hat{c}}(\sqrt{\pi} \lambda)^{4} \delta^{2}\left(k_{1, \perp}^{\prime}-\tilde{k}_{1, \perp}\right) \delta\left(k_{1-}^{\prime}-\tilde{k}_{1-}\right) \delta\left(k_{1+}^{\prime}-\tilde{k}_{1+}\right)(2 \pi)^{4} \\
& \exp \left\{-\lambda^{2}\left[\left(k_{1, \perp}^{\prime}+\tilde{k}_{1, \perp}\right)^{2}+\left(k_{1-}^{\prime}+\tilde{k}_{1-}\right)^{2}+\left(k_{1+}^{\prime}+\tilde{k}_{1+}\right)^{2}\right] / 4\right\} \\
= & C \delta_{c \hat{c}}(\sqrt{\pi} \lambda)^{4} \delta^{2}\left(k_{1, \perp}^{\prime}-\tilde{k}_{1, \perp}\right) \delta\left(k_{1-}^{\prime}-\tilde{k}_{1-}\right) \delta\left(k_{1+}^{\prime}-\tilde{k}_{1+}\right)(2 \pi)^{4} \\
& \exp \left\{-\lambda^{2}\left[\left(k_{1, \perp}^{\prime}\right)^{2}+\left(k_{1-}^{\prime}\right)^{2}+\left(k_{1+}^{\prime}\right)^{2}\right]\right\}
\end{aligned}
$$

with $Y^{(+)}=y^{\prime}+\tilde{y}$ and $Y^{(-)}=y^{\prime}-\tilde{y}$. $C$ is a constant to be determine from a suitable normalization condition.

Now we are at the point where the normalization of $d$, respectively $D$, has to be discussed. To do so we start from the energy associated with the quadratic part of the Yang-Mills Lagrangian,

$$
\mathcal{L}=-\frac{1}{2}\left(\partial_{\mu} A_{\nu}^{a}-\partial_{\nu} A_{\mu}^{a}\right) \partial^{\mu} A_{a \nu}
$$

which should be equivalent to some integral of the form

$$
E=\int \frac{d^{4} p}{(2 \pi)^{2}} \quad \ldots d(p, p)
$$

with a one-particle, not yet normalized, density matrix $d(p, p)$. ¿From Eq. (42) we get

$$
E=\frac{1}{2} \int d^{3} x\left(\left(\partial^{0} A^{b j}(x)\right)^{2}-\left(\partial^{j} A^{b 0}(x)\right)^{2}+\left(\partial^{i} A^{b j}(x)\right)\left(\partial^{i} A^{b j}(x)\right)-\left(\partial^{i} A^{b j}(x)\right)\left(\partial^{j} A^{b i}(x)\right)\right)
$$

Substituting

$$
A^{b \mu}(x) A^{b \nu}(x) \rightarrow \int \frac{d^{4} p}{(2 \pi)^{4}} \int \frac{d^{4} \tilde{p}}{(2 \pi)^{4}} e^{\mathrm{i}(p-\tilde{p}) \cdot x} d^{\mu \nu}(b, b ; p ; \tilde{p})
$$

we get

$$
E=\frac{1}{2} \int \frac{d^{4} p d \tilde{p}^{0}}{(2 \pi)^{5}}\left(\left(p^{0} \tilde{p}^{0}+(\vec{p})^{2}\right) d^{j j}\left(b, b ; p^{0}, \vec{p} ; \tilde{p}^{0}, \vec{p}\right)-(\vec{p})^{2} d^{00}\left(b, b ; p^{0}, \vec{p} ; \tilde{p}^{0}, \vec{p}\right)-p^{i} \tilde{p}^{j} d^{j i}\left(b, b ; p^{0}, \vec{p} ; \tilde{p}^{0}, \vec{p}\right)\right)
$$

For simplicity we choose the gauge $p_{\mu} A^{\mu}=0$, allowing the last term to be re-expressed in the form $p^{0} \tilde{p}^{0} d^{00}$. Our model assumptions Eq. (41) imply that we only need the diagonal matrix elements of $D$. To fix it we require that Eq. (46) reproduces $\bar{p}^{0}$ for a plane wave gluon with 4 momentum $\bar{p}^{\mu}$, i.e. we impose:

$$
\bar{p}^{0}=\frac{1}{2} \int \frac{d^{4} p d \tilde{p}^{0}}{(2 \pi)^{5}}\left(\left(p^{0} \tilde{p}^{0}+(\vec{p})^{2}\right) d_{\bar{p}}^{j j}\left(b, b ; p^{0}, \vec{p}, \tilde{p}^{0}, \vec{p}\right)-\left(p^{0} \tilde{p}^{0}+(\vec{p})^{2}\right) d_{\bar{p}}^{00}\left(b, b ; p^{0}, \vec{p}, \tilde{p}^{0}, \vec{p}\right)\right)
$$

for a density matrix of the form

$$
d_{\bar{p}}^{\mu \nu}\left(b, b ; p^{0}, \vec{p}, \tilde{p}^{0}, \vec{p}\right)=\delta_{\mu k} \delta_{\nu k}(2 \pi)^{4} \delta^{4}(p-\bar{p}) f\left(p^{0}, \tilde{p}^{0}, \vec{p}\right)
$$

The solution for $f$ is obviously

$$
f\left(p^{0}, \tilde{p}^{0}, \vec{p}\right)=\delta\left(p^{0}-\tilde{p}^{0}\right) \frac{p^{0} 4 \pi}{\left(p^{0}\right)^{2}+(\vec{p})^{2}}
$$


This motivates our assumption that the diagonal matrix elements of our $d$ are related to those of the properly normalized density matrix $D$ by the factor (setting $\delta(0)=T$ )

$$
d^{\mu \nu}\left(b, b ; p^{0}, \vec{p}, p^{0}, \vec{p}\right)=T \frac{p^{0} 4 \pi}{\left(p^{0}\right)^{2}+(\vec{p})^{2}} \quad D^{\mu \nu}\left(b, b ; p^{0}, \vec{p}, p^{0}, \vec{p}\right) V T,
$$

where $D$ is according to Eq. (41) in our model given by

$$
D_{\nu}^{\mu}\left(b, b ; p^{0}, \vec{p}, p^{0}, \vec{p}\right)=\left.\frac{16}{V T} \pi^{2} \lambda^{4} \exp \left\{-\lambda^{2}\left[\left(p_{\perp}\right)^{2}+\left(p_{-}\right)^{2}+\left(p_{+}\right)^{2}\right]\right\} \frac{1}{2} \delta_{\mu \nu}\right|_{\mu, \nu \neq \perp} .
$$

Here the superscript " $\perp$ " indicates that the Kronecker symbol contributes only for the transverse directions $\mu, \nu=1,2$, and the factor $\frac{1}{2}$ encodes the unpolarized nature of the gluons in the target nucleus. The normalization of the trace of the density matrix demands that

$$
V T \int \frac{d^{4} p}{(2 \pi)^{4}} D_{\mu}^{\mu}\left(b, b ; p^{0}, \vec{p}, p^{0}, \vec{p}\right)=1
$$

for a one-gluon state.

For a many-gluon state this should be normalized to the total number of gluons which is an ill-defined quantity. We therefore choose to substitute for this general case $E=\bar{p}^{0}$ by the total energy of the gluons $E_{G}\left(Q_{s}^{2}\right)$ in nucleus 1 , at the transverse scale with which this nucleus is resolved, which is the saturation scale.

Combining everything the density matrix in the boosted, previously unpopulated region of phase space is

$$
\begin{aligned}
\left.\frac{1}{2} \delta_{\mu \nu}\right|_{\mu, \nu \neq \perp} \cdot D_{1 \hat{1}}\left(c, \hat{c} ; k_{1}^{0}, \vec{k}_{1}, \hat{k}_{1}^{0}, \overrightarrow{\hat{k}}_{1}\right)=\int \frac{d^{4} \tilde{k}_{1}}{(2 \pi)^{4}} \int \frac{d^{4} k_{1}^{\prime}}{(2 \pi)^{4}}(2 \pi)^{4} \delta^{4}\left(k_{1}^{\prime}-\tilde{k}_{1}\right) \frac{1}{V T} \\
\mathcal{W}_{1 \hat{1}, \tilde{1} \tilde{1}} \frac{4 \pi E_{G}\left(Q_{s}^{2}\right) T}{\left(\tilde{k}_{1}^{0}\right)^{2}+\left(\overrightarrow{\tilde{k}}_{1}\right)^{2}} D_{\mu \nu}\left(b, b ; k_{1}^{\prime}, \tilde{k}_{1}\right) V T
\end{aligned}
$$

with

$$
f_{a b c} f_{a b \hat{c}} \epsilon\left(k_{1}^{\prime}\right)_{-} \epsilon^{*}\left(k_{1}^{\prime}\right)_{-} f_{a b c} f_{a b \hat{c}} \rightarrow N_{c} \delta_{c \hat{c}} \frac{1}{3}
$$

The factor $\frac{1}{3}$ is motivated by the assumption that in a nucleus at rest (the target nucleus 1 ) all gluons are so highly virtual that the transverse and longitudinal polarization components contribute equally. The gauge degrees of freedom, of course, do not contribute.

Combining all our results we get for the one particle density matrix in the final state phase space region (substituting $\tilde{k}_{1}$ by $p$ for notational simplicity):

$$
\begin{aligned}
D_{1, \hat{1}}= & \int \frac{d^{4} p}{(2 \pi)^{4}} \frac{4 \pi E_{G}\left(Q_{s}^{2}\right) 16 \pi^{2} \lambda^{4} N_{c} \delta_{c \hat{c}}}{3 V^{2}\left(\left(p^{0}\right)^{2}+(\vec{p})^{2}\right)} \exp \left\{-\lambda^{2}\left[\left(p_{\perp}^{2}+p_{-}^{2}+p_{+}^{2}\right]\right\}\right. \\
& \frac{(2 \pi)^{3} \delta^{2}\left(k_{1 \perp}-\hat{k}_{1 \perp}\right)}{\sqrt{2 k_{1+} \hat{k}_{1+}}} \theta\left(\hat{k}_{1+}-p_{+}\right) \theta\left(k_{1+}-p_{+}\right) \delta\left(p_{-}-k_{1-}\right) \delta\left(p_{-}-\hat{k}_{1-}\right) \\
& 8 k_{1+} \hat{k}_{1+}\left(1+\frac{k_{1, \perp} \cdot \hat{k}_{1, \perp}}{2 k_{1} \cdot \hat{k}_{1}}\right) \frac{1}{\left(k_{1+}-p_{+}+\mathrm{i} \epsilon\right)\left(\hat{k}_{1+}-p_{+}-\mathrm{i} \epsilon\right)} \mathrm{E}_{1}\left(\frac{\left(k_{1 \perp}-p_{\perp}\right)^{2}}{Q_{s}^{2}}\right)
\end{aligned}
$$

In order to evaluate this expression, we note that we are interested in the scattering of gluons into states with $k_{1+}, \hat{k}_{1+} \gg p_{+} \sim \mathcal{O}\left(\lambda^{-1}\right)$. We can then neglect the $p_{+}$dependence of the two denominators, drop the step functions, and obtain:

$$
\begin{aligned}
16 \pi^{2} & \int \frac{d^{4} p}{(2 \pi)^{4}} \frac{e^{-\lambda^{2}\left[p_{\perp}^{2}+p_{-}^{2}+p_{+}^{2}\right]}}{p_{\perp}^{2}+p_{-}^{2}+p_{+}^{2}} \delta\left(p_{-}-k_{1-}\right) \delta\left(p_{-}-\hat{k}_{1-}\right) \mathrm{E}_{1}\left(\frac{\left(k_{1 \perp}-p_{\perp}\right)^{2}}{Q_{s}^{2}}\right) \\
& =\frac{1}{\pi^{2}} \delta\left(k_{1-}-\hat{k}_{1-}\right) \int_{\lambda^{2}}^{\infty} d \xi^{2} \int_{-\infty}^{\infty} d p_{+} \int_{-\infty}^{\infty} d^{2} p_{\perp} e^{-\xi^{2}\left(p_{+}^{2}+p_{\perp}^{2}+k_{1-}^{2}\right)} \mathrm{E}_{1}\left(\frac{\left(k_{1 \perp}-p_{\perp}\right)^{2}}{Q_{s}^{2}}\right) \\
& =\frac{2}{\pi^{3 / 2}} \delta\left(k_{1-}-\hat{k}_{1-}\right) \int_{\lambda}^{\infty} d \xi \int_{0}^{1} \frac{d u}{u} \int^{2} p_{\perp} e^{-\xi^{2}\left(p_{\perp}^{2}+k_{1-}^{2}\right)} e^{-\left(k_{1 \perp-}-p_{\perp}\right)^{2} /\left(Q_{s}^{2} u\right)} \\
& =\frac{2}{\sqrt{\pi}} \delta\left(k_{1-}-\hat{k}_{1-}\right) \int_{\lambda}^{\infty} d \xi e^{-\xi^{2} k_{1-}^{2}} \int_{0}^{1} d u \frac{Q_{s}^{2}}{u Q_{s}^{2} \xi^{2}+1} \exp \left(-\frac{\xi^{2} k_{1 \perp}^{2}}{u Q_{s}^{2} \xi^{2}+1}\right)
\end{aligned}
$$


We now have traded 3 integrals (over $p_{+}$and $p_{\perp}$ ) for two integrals (over $\xi$ and $u$ ). This may not seem like much progress, but it turns out that the integral over $u$ can be done after the substitution $s=\xi^{2} Q_{s}^{2} /\left(u \xi^{2} Q_{s}^{2}+1\right)$ :

$$
\begin{aligned}
\int_{0}^{1} d u \frac{1}{u Q_{s}^{2} \xi^{2}+1} \exp \left(-\frac{\xi^{2} k_{1 \perp}^{2}}{u Q_{s}^{2} \xi^{2}+1}\right) & =\int_{\frac{Q_{s}^{2} \xi^{2}}{Q_{s}^{2} \xi^{2}+1}}^{\xi^{2} Q_{s}^{2}} \frac{d s}{s^{2}} \frac{s}{\xi^{2} Q_{s}^{2}} e^{-s k_{1 \perp}^{2} / Q_{s}^{2}} \\
& =\frac{1}{Q_{s}^{2} \xi^{2}}\left[E_{1}\left(\frac{k_{1 \perp}^{2} \xi^{2}}{Q_{s}^{2} \xi^{2}+1}\right)-E_{1}\left(k_{1 \perp}^{2} \xi^{2}\right)\right] .
\end{aligned}
$$

We finally substitute $\xi \rightarrow \lambda \xi$ in the remaining integration and obtain for the expression (55)

$$
\begin{aligned}
D_{1, \hat{1}}= & \frac{(4 \pi)^{4} \lambda^{3} E_{G} N_{c} \delta_{c \hat{c}}}{3 V^{2}} \frac{\delta^{2}\left(k_{1 \perp}-\hat{k}_{1 \perp}\right) \delta\left(k_{1-}-\hat{k}_{1-}\right)}{\sqrt{2 k_{1+} \hat{k}_{1+}}}\left(1+\frac{k_{1, \perp} \cdot \hat{k}_{1, \perp}}{2 k_{1} \cdot \hat{k}_{1}}\right) \\
& \times \frac{2}{\sqrt{\pi}} \int_{1}^{\infty} \frac{d \xi}{\xi^{2}} e^{-\lambda^{2} k_{1-}^{2} \xi^{2}}\left[E_{1}\left(\frac{\lambda^{2} k_{1 \perp}^{2} \xi^{2}}{\lambda^{2} Q_{s}^{2} \xi^{2}+1}\right)-E_{1}\left(\lambda^{2} k_{1 \perp}^{2} \xi^{2}\right)\right] .
\end{aligned}
$$

This expression describes the density matrix of the liberated gluons, which are scattered out of the target nucleus 1 by the quasi-real gluons of the fast moving projectile nucleus 2 . We note that the density matrix is diagonal in the momentum components $k_{1-}$ and $k_{1 \perp}$, but not in the component $k_{1+}$. The physical reason for this asymmetric behavior is that the projectile nucleus is moving very fast in the $x_{-}$direction. This implies that the distribution of its gluons in $k_{+}$is very broad and leads to interference of excitation amplitudes of gluons from the target nucleus into final states with different values of $k_{1+}$.

\section{THE DECOHERENCE TIME}

We now calculate the ratio (9) for the density matrix from Eq. (58). we define

$$
F\left(k_{1-}, k_{1 \perp}\right)=\int_{1}^{\infty} \frac{d \xi}{\xi^{2}} e^{-\lambda^{2} k_{1-}^{2} \xi^{2}}\left[E_{1}\left(\frac{\lambda^{2} k_{1 \perp}^{2} \xi^{2}}{\lambda^{2} Q_{s}^{2} \xi^{2}+1}\right)-E_{1}\left(\lambda^{2} k_{1 \perp}^{2} \xi^{2}\right)\right]
$$

Because any constant factors will drop out of the ratio (9), it is sufficient to consider the $k$-dependent part of $D_{1, \hat{1}}$, which we call the reduced density matrix:

$$
\mathcal{D}_{1, \hat{1}}=\frac{F\left(k_{1-}, k_{1 \perp}\right)}{\sqrt{k_{1+} \hat{k}_{1+}}} \delta\left(k_{1-}-\hat{k}_{1-}\right) \delta^{2}\left(k_{1 \perp}-\hat{k}_{1 \perp}\right),
$$

where we have neglected the factor $(1+\ldots)$ deriving from the polarization sum, which is of order unity. We obtain:

$$
\begin{aligned}
\operatorname{Tr} \mathcal{D} & =\frac{V T}{(2 \pi)^{4}} \delta\left(0_{-}\right) \delta^{2}\left(0_{\perp}\right) \int \frac{d k_{1+}}{k_{1+}} \int_{0}^{\infty} d k_{1-} \int_{-\infty}^{\infty} d^{2} k_{1 \perp} F\left(k_{1-}, k_{1 \perp}\right) \\
& =\frac{V T}{(2 \pi)^{4}} \delta\left(0_{-}\right) \delta^{2}\left(0_{\perp}\right) \int \frac{d k_{1+}}{k_{1+}} \frac{\pi^{3 / 2}}{4 \lambda} Q_{s}^{2}
\end{aligned}
$$

The details of the integration can be found in appendix A. For $\operatorname{Tr} \mathcal{D}^{2}$ we obtain

$$
\begin{aligned}
\operatorname{Tr}^{2}= & \left(\frac{V T}{(2 \pi)^{4}}\right)^{2} \int d^{4} k \int d^{4} \hat{k} \frac{1}{k_{1+} \hat{k}_{1+}} \\
& F\left(k_{1-}, k_{\perp}\right) \delta\left(k_{1-}-\hat{k}_{1-}\right) \delta^{2}\left(k_{1 \perp}-\hat{k}_{1 \perp}\right) F\left(k_{1-}, k_{\perp}\right) \delta\left(k_{1-}-\hat{k}_{1-}\right) \delta^{2}\left(k_{1 \perp}-\hat{k}_{1 \perp}\right) \\
= & \left(\frac{V T}{(2 \pi)^{4}}\right)^{2} \int \frac{d k_{1+}}{k_{1+}} \int \frac{d \hat{k}_{1+}}{\hat{k}_{1+}} \delta\left(0_{-}\right) \delta^{2}\left(0_{\perp}\right) \int_{0}^{\infty} d k_{1-} \int_{-\infty}^{\infty} d^{2} k_{1 \perp}\left(F\left(k_{1-}, k_{\perp}\right)\right)^{2}
\end{aligned}
$$

Which can be simplyfied using the identity (see Appendix A)

$$
\int_{-\infty}^{\infty} d k_{1 \perp}^{2} E_{1}\left(a k_{1 \perp}^{2}\right) E_{1}\left(b k_{1 \perp}^{2}\right)=\frac{1}{b} \ln \frac{a+b}{a}+\frac{1}{a} \ln \frac{a+b}{b}
$$




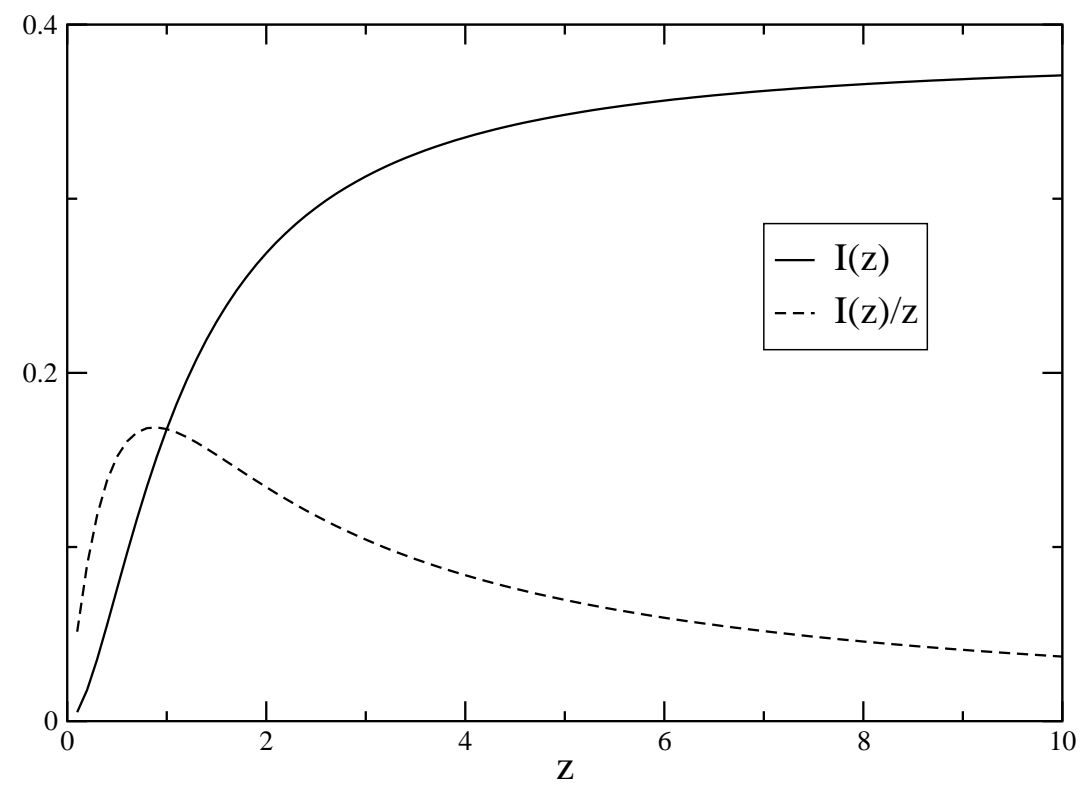

FIG. 3: The function $I(z)$ and the ratio $I(z) / z$

which leads to

$$
\begin{gathered}
\operatorname{Tr} \mathcal{D}^{2}=\left(\frac{V T}{(2 \pi)^{4}}\right)^{2} \int \frac{d k_{1+}}{k_{1+}} \int \frac{d \hat{k}_{1+}}{\hat{k}_{1+}} \delta\left(0_{-}\right) \delta^{2}\left(0_{\perp}\right) \frac{\pi \sqrt{\pi}}{2 \lambda} Q_{s}^{2} \int_{1}^{\infty} \frac{d \xi}{\xi^{2}} \int_{1}^{\infty} \frac{d \chi}{\chi^{2}} \frac{1}{\sqrt{\xi^{2}+\chi^{2}}} \\
\sum_{i, j=1}^{2}(-1)^{i+j}\left[\frac{1}{b_{j}} \ln \frac{a_{i}+b_{j}}{a_{i}}+\frac{1}{a_{i}} \ln \frac{a_{i}+b_{j}}{b_{j}}\right]
\end{gathered}
$$

with

$$
\begin{aligned}
& a_{1}=\frac{\lambda^{2} Q_{s}^{2} \xi^{2}}{\lambda^{2} Q_{s}^{2} \xi^{2}+1} \quad, \quad a_{2}=\lambda^{2} Q_{s}^{2} \xi^{2} \\
& b_{1}=\frac{\lambda^{2} Q_{s}^{2} \chi^{2}}{\lambda^{2} Q_{s}^{2} \chi^{2}+1} \quad, \quad b_{2}=\lambda^{2} Q_{s}^{2} \chi^{2}
\end{aligned}
$$

We now define the integral

$$
I\left(\lambda Q_{s}\right)=\int_{1}^{\infty} \frac{d \xi}{\xi^{2}} \int_{1}^{\infty} \frac{d \chi}{\chi^{2}} \frac{1}{\sqrt{\xi^{2}+\chi^{2}}} \sum_{i, j=1}^{2}(-1)^{i+j}\left[\frac{1}{b_{j}} \ln \frac{a_{i}+b_{j}}{a_{i}}+\frac{1}{a_{i}} \ln \frac{a_{i}+b_{j}}{b_{j}}\right],
$$

which approaches zero for $\lambda Q_{s} \rightarrow 0$ and approaches for asymptotically large argument the limit $I(\infty)=\frac{4}{3}(\sqrt{2}-$ $1) \ln 2 \approx 0.3828$. One can see the latter as follows: $\lambda Q_{s}$ is much larger than unity and also $\xi, \chi \geq 1$. Therefore $a_{2} \gg a_{1} \sim 1$ and $b_{2} \gg b_{1} \sim 1$ and the contribution with $i=j=1$ dominates. In this limit, the square bracket assumes a value of about $2 \ln 2$. Substituting this value into the integral (68), we obtain the asymptotic limit mentioned above. The exact form of $I\left(\lambda Q_{s}\right)$ is given in Fig. 3

The degree of decoherence is given by the ratio

$$
\frac{\operatorname{Tr} \mathcal{D}^{2}(\text { total })}{(\operatorname{Tr} \mathcal{D}(\text { total }))^{2}}=\frac{\operatorname{Tr}[\mathcal{D}(\text { target })+\mathcal{D}]^{2}}{(\operatorname{Tr}[\mathcal{D}(\text { target })+\mathcal{D}])^{2}}
$$

where $\mathcal{D}$ (target) is the density matrix of that part of the phase space which contained the original target. We are interested in the situation that most gluons of nucleus 1 have already undergone a hard scattering, i.e. $T$ must be large enough, typically of order $\geq 0.1 \mathrm{fm} / \mathrm{c}$. Then we can disregard $\mathcal{D}$ (target).

Thus we finally obtain for the ratio which characterizes the degree of decoherence after the hard gluon scattering

$$
\frac{\operatorname{Tr} \mathcal{D}^{2}}{(\operatorname{Tr} \mathcal{D})^{2}}=\frac{\pi \sqrt{\pi}}{2 \lambda} \frac{16 \lambda^{2} I\left(\lambda Q_{s}\right) Q_{s}^{2}}{\pi^{3} Q_{s}^{4} \delta^{2}\left(0_{\perp}\right) \delta\left(0_{-}\right)}=\frac{8 I\left(\lambda Q_{s}\right) \lambda}{\pi \sqrt{\pi} Q_{s}^{2}} \cdot \frac{\pi}{\lambda^{2}} \cdot \frac{1}{T}
$$


With the "observation time" $\delta\left(0_{-}\right)=T$ and $\delta^{2}\left(0_{\perp}\right)=\lambda^{2} / \pi$, which is the correct normalization for eq.(152), because

$$
\delta^{4}(0)=\frac{V T}{(2 \pi)^{4}} D_{\mu}^{\mu}(0)=\frac{\lambda^{4}}{\pi^{2}} .
$$

We now define the decoherence time as the value of $T$ for which the ratio (68) becomes equal to $1 / e$. This gives

$$
\tau_{\mathrm{deco}}=\frac{8 e I\left(\lambda Q_{s}\right)}{\sqrt{\pi} Q_{s} \lambda} \cdot \frac{1}{Q_{s}}
$$

For realistic values $\left(\lambda=0.3 \mathrm{fm}, Q_{s}=1 \mathrm{GeV}\right)$ the first factor is numerically of order unity (more precisely it is 1.865 ) for these values) and we can conclude

$$
\tau_{\text {deco }} \sim \frac{1}{Q_{s}}
$$

This is our main result. The decoherence time is of the order of $0.3-0.4 \mathrm{fm} / \mathrm{c}$, and thus large enough to neglect $\mathcal{D}$ (target) in Eq. (67), and drops with increasing saturation scale $Q_{s}$, i.e. with increasing collision energy. However, $Q_{s}$ increases so slowly with $s$ that the latter effect is rather marginal. We use $\lambda Q_{s}=1.5$ and think that the uncertainty in this value is not large. Nevertheless, we want to point out that even if one varied this product by a factor of two, the ratio $I\left(\lambda Q_{s}\right) / \lambda Q_{s}$ would change only marginaly and (71) stayed valid. (For $\lambda Q_{s}$ much smaller than one our description stops making sense.)

\section{CONCLUSIONS}

We have calculated the characteristic decoherence time in high energy heavy ion collisions due to gluon scattering. We find that this time is substantially shorter than $1 \mathrm{fm} / c$. This result furnishes the remaining logical link in our argument that decoherence alone can explain a substantial part of the entropy production during the earliest phase of a heavy ion collison. We note that the decohered partonic state of the colliding nuclei is not yet thermally equilibrated. Additional interactions among the decohered quanta, such as those invoked in the bottom-up scenario of equilibration [12] or modified versions of it [13, 14] are required to achieve full equilibration.

\section{Acknowledgments:}

One of us (BM) gratefully acknowledges support from the Alexander von Humboldt Foundation in the form of a Senior Scientist Award. This work was also supported in part by grants from the Office of Science of the U. S. Department of Energy and from the BMBF.

\section{APPENDIX A}

We here evaluate the integral appearing in eq. (60):

$$
\begin{aligned}
I^{\prime} & =\int_{0}^{\infty} d k_{1-} \int_{-\infty}^{\infty} d^{2} k_{1 \perp} \int_{1}^{\infty} \frac{d \xi}{\xi^{2}} e^{-\lambda^{2} k_{1-}^{2} \xi^{2}}\left[E_{1}\left(\frac{\lambda^{2} k_{1 \perp}^{2} \xi^{2}}{\lambda^{2} Q_{s}^{2} \xi^{2}+1}\right)-E_{1}\left(\lambda^{2} k_{1 \perp}^{2} \xi^{2}\right)\right] \\
& =\frac{\sqrt{\pi}}{2 \lambda} \int_{-\infty}^{\infty} d^{2} k_{1 \perp} \int_{1}^{\infty} \frac{d \xi}{\xi^{3}}\left[E_{1}\left(\frac{\lambda^{2} k_{1 \perp}^{2} \xi^{2}}{\lambda^{2} Q_{s}^{2} \xi^{2}+1}\right)-E_{1}\left(\lambda^{2} k_{1 \perp}^{2} \xi^{2}\right)\right] \\
& =\frac{\sqrt{\pi}}{2 \lambda} \int_{1}^{\infty} \frac{d \xi}{\xi^{3}}\left[\frac{\lambda^{2} Q_{s}^{2} \xi^{2}+1}{\lambda^{2} \xi^{2}}-\frac{1}{\lambda^{2} \xi^{2}}\right] \\
& =\frac{\sqrt{\pi}}{2 \lambda} Q_{s}^{2} \frac{1}{2}
\end{aligned}
$$

where we used the relation

$$
\int_{0}^{\infty} d z E_{1}(z)=\int_{0}^{\infty} d z \int_{z}^{\infty} \frac{d t}{t} e^{-t}=\int_{0}^{\infty} \frac{d t}{t} \int_{0}^{t} d z e^{-t}=\int_{0}^{\infty} d t e^{-t}=1
$$


We also derive the identity (62):

$$
\begin{aligned}
\int_{-\infty}^{\infty} d^{2} k_{1 \perp} E_{1}\left(a k_{1 \perp}\right) E_{1}\left(b k_{1 \perp}\right) & =\frac{\pi}{2} \int_{0}^{\infty} d k_{1 \perp}^{2} \int_{1}^{\infty} \frac{d t}{t} e^{-a k_{1 \perp}^{2} t} \int_{1}^{\infty} \frac{d s}{s} e^{-b k_{1 \perp}^{2} s} \\
& =\frac{\pi}{2} \int_{1}^{\infty} \frac{d t}{t} \int_{1}^{\infty} \frac{d s}{s} \frac{1}{a t+b s} \\
& =\frac{\pi}{2} \int_{1}^{\infty} \frac{d t}{t}\left[\frac{1}{a t} \ln \left(\frac{s}{a t+b s}\right)\right]_{1}^{\infty} \\
& =\frac{\pi}{2 a} \int_{1}^{\infty} \frac{d t}{t^{2}}[\ln (a t+b)-\ln (b)] \\
& =\frac{\pi}{2 b} \int_{a / b}^{\infty} \frac{d y}{y^{2}} \ln (y+1) \\
& =\frac{\pi}{2 b}\left[\ln \left(\frac{y}{y+1}\right)-\frac{1}{y} \ln (y+1)\right]_{a / b}^{\infty} \\
& =\frac{\pi}{2}\left[\frac{1}{b} \ln \left(\frac{a+b}{a}\right)+\frac{1}{a} \ln \left(\frac{a+b}{b}\right)\right]
\end{aligned}
$$

[1] Quark-Gluon Plasma (New Discoveries at RHIC: A case for the strongly interacting quark-gluon plasma), ed. by D. Rischke and G. Levin, Nucl. Phys. A 750, 1 (2005).

[2] I. Arsene et al. [BRAHMS Collaboration], Nucl. Phys. A 757, 1 (2005); B. B. Back et al. [PHOBOS Collaboration], Nucl. Phys. A 757, 28 (2005); J. Adams et al. [STAR Collaboration], Nucl. Phys. A 757, 102 (2005); K. Adcox et al. [PHENIX Collaboration], Nucl. Phys. A 757, 184 (2005).

[3] B. Müller and A. Schäfer, arXiv:hep-ph/0306309

[4] L. V. Gribov, E. M. Levin and M. G. Ryskin, Phys. Rept. 100, 1 (1983); A. H. Mueller and J. w. Qiu, Nucl. Phys. B 268, 427 (1986).

[5] H. T. Elze and P. A. Carruthers, arXiv:hep-ph/9409248 see also H. T. Elze, arXiv:quant-ph/9710063 Nucl. Phys. Proc. Suppl. 39BC (1995) 169, arXiv:hep-ph/9407377 Phys. Lett. B 369 (1996) 295, arXiv:hep-th/9406085

[6] C. Anastopoulos, Int. J. Theor. Phys. 41 (2002) 1573.

[7] J. J. Halliwell, Phys. Rev. D 58, 105015 (1998).

[8] D. Hooper, D. Morgan and E. Winstanley, Phys. Lett. B 609, 206 (2005); G. Barenboim and N. E. Mavromatos, JHEP 0501, 034 (2005); S. L. Adler, J. Phys. A 38, 2729 (2005).

[9] H. Weigert, Prog. Part. Nucl. Phys. 55, 461 (2005).

[10] Y. V. Kovchegov and A. H. Mueller, Nucl. Phys. B 529, 451 (1998).

[11] Y. V. Kovchegov, Nucl. Phys. A 692, 557 (2001).

[12] R. Baier, A. H. Mueller, D. Schiff and D. T. Son, Phys. Lett. B 502, 51 (2001).

[13] D. Bodeker, JHEP 0510, 092 (2005) arXiv:hep-ph/0508223.

[14] A. H. Mueller, A. I. Shoshi and S. M. H. Wong, arXiv:hep-ph/0512045 